\title{
DUALITY OF ALBANESE AND PICARD 1-MOTIVES
}

\author{
NIRANJAN RAMACHANDRAN
}

\begin{abstract}
We define Albanese and Picard 1-motives of smooth (simplicial) schemes over a perfect field. For smooth proper schemes, these are the classical Albanese and Picard varieties. For a curve, these are the homological 1-motive of Lichtenbaum and the motivic $H^{1}$ of Deligne. This paper proves a conjecture of Deligne about providing an algebraic description, via 1-motives, of the first homology and cohomology groups of a complex algebraic variety. (L. Barbieri-Viale and V. Srinivas have also proved this independently.) It also contains a purely algebraic proof of Lichtenbaum's conjecture that the Albanese and the Picard 1-motives of a (simplicial) scheme are dual. This gives a new proof of an unpublished theorem of Lichtenbaum that Deligne's 1-motive of a curve is dual to Lichtenbaum's 1-motive.
\end{abstract}

\section{Revised version}

\section{INTRODUCTION}

Let $k$ be a perfect field. Two classical invariants of any smooth projective variety $X$ over $k$ are the abelian varieties $A l b(X)$ (the Albanese variety), and $\operatorname{Pic}(X)$ (the Picard variety) [5]. It is a classical observation of W. L. Chow, J. Igusa, and A. Weil that $A l b(X)$ and $\operatorname{Pic}(X)$ are naturally dual.

In this paper, we extend this classical result, using 1-motives, to arbitrary schemes over $k$. P. Deligne's technique [6] $\S 6.3$ of defining a cohomological invariant for arbitrary varieties consists of defining it for simplicial schemes $X_{\bullet}$ (this is the complement of a simplicial divisor with normal crossings $E_{\bullet}$ in a smooth projective simplicial scheme $X_{\bullet}^{\prime}$ ) and using smooth hypercoverings to define it for varieties (see $\delta$ (]). For any such scheme $X_{\bullet}$, we will define the Albanese 1-motive $M_{1}\left(X_{\bullet}\right)$ and the Picard 1-motive $M^{1}\left(X_{\bullet}\right)$. Our construction of the covariant functor $M_{1}$ is based on the generalized Albanese variety of Rosenlicht-Lang-Serre. The construction of the contravariant functor $M^{1}$ is based upon the Picard scheme of a simplicial scheme.

Our main result (Theorem 5.1) is that, for any $X_{\bullet}$, the 1-motives $M^{1}$ and $M_{1}$ are naturally dual. This was conjectured by S. Lichtenbaum. When $X_{\bullet}=X_{\bullet}^{\prime}$ is a constant scheme corresponding to a smooth projective variety $V$ (i.e., each $X_{i}=V$ ), Theorem 5.1 reduces to the classical duality of $A l b(V)$ and $\operatorname{Pic}(V)$.

Date: 30 September 2000.

Supported by NSF and the Alfred P. Sloan Doctoral Dissertation Fellowship, 1995-96. 
For any curve $C$, Deligne has defined a 1-motive $H_{m}^{1}(C)(1)$ and obtained, via the realization functors on one-motives, the $\ell$-adic cohomology, De Rham cohomology etc., of $C$ from $H_{m}^{1}(C)(1)$. Lichtenbaum 21] showed that a 1motive appears, rather naturally, in the Suslin complex of the curve $C$. This led him to define $h_{1}(C)$, the homological 1-motive of $C$. The homological 1-motive of a smooth curve is readily seen to be a generalized Jacobian of M. Rosenlicht 32, 33.

Associated with any curve $C$ is a canonical smooth simplicial scheme $Z$. (§); [6] $\S 10.3)$. We show that $M_{1}\left(Z_{\bullet}\right)=h_{1}(C)$ and $M^{1}\left(Z_{\bullet}\right)=H_{m}^{1}(C)(1)$. As a consequence of Theorem 5.1, we obtain a new proof of an unpublished theorem of Lichtenbaum (Theorem 6.8): the 1-motive $h_{1}(C)$ is naturally dual to the 1-motive $H_{m}^{1}(C)(1)$.

Let $X_{\bullet}$ be a scheme (as above) over $\mathbb{C}$. We also show that the Hodge realization of $M^{1}\left(X_{\bullet}\right)$ is the mixed Hodge structure $H^{1}\left(X_{\bullet} ; \mathbb{Z}(1)\right)$. It follows from the duality theorem (Theorem 5.1) that the Hodge realization of $M_{1}\left(X_{\bullet}\right)$ is the mixed Hodge structure on $H_{1}\left(X_{\bullet}\right) /$ tor sion. This settles the conjecture of Deligne for $I\left(H^{1}\left(X_{\bullet}\right)(1)\right)(\S 10.4 .1$ of [6]). Results about the other realizations of these 1-motives will appear elsewhere.

Weak Lefschetz-type theorems are available for varieties which are either non-singular quasi-projective or affine (14 pp. 23-24); it is also remarked in (loc. cit) that such theorems do not hold in general for arbitrary varieties. Therefore, the study of the motivic $H^{1}$ and $H_{1}$ for general varieties does not seem to reduce to the case of curves. This means that the results obtained here about the motivic $H^{1}$ and $H_{1}$ are perhaps of interest. Related work in this direction includes [27], [12], [1], [2], [11], [22], and [30]. I should mention that 30 generalizes to $H^{n}$ (for $n>1$ ) 1 the results obtained here for $H^{1}$. But the Albanese 1-motive and the duality theorem are not touched there. Recently L. Barbieri-Viale and V. Srinivas [2] (announced in [1]) have independently proved Theorems 3.2 and 4.4. One (the essential) difference between their work and ours is that their Albanese 1-motive is defined to be the dual of the Picard 1-motive. Another is that they define their invariants only in characteristic zero, whereas we do so for an arbitrary perfect field (The restriction to perfect fields is forced by Theorem 3.4).

0.1. Notations. We use the following conventions.

$S=$ Spec $k$, the spectrum of a perfect field $k$.

The characteristic of $k$ is denoted by $p$.

$\bar{S}=$ Spec $\bar{k}$, the spectrum of an algebraic closure $\bar{k}$ of $k$.

$\mathrm{g}=\operatorname{Gal}(\bar{k} / k)$, the Galois group of $\bar{k}$ over $k$.

We consider only separated schemes $X$ over $S$. Such schemes are supposed to be of finite type over $S$ unless otherwise mentioned. We use $\bar{X}$ to denote the $\bar{S}$-scheme $X \times_{S} \bar{S}$ defined by the $S$-scheme $X$.

$\tilde{X}:=$ the normalization of $X$.

\footnotetext{
${ }^{1}$ In [3], the conjecture of Deligne is proved up to isogeny for $H^{n}(V)$; here, our theorem proves the integral version of the conjecture for $H^{1}$.
} 
$X^{\text {red }}:=$ the maximal open and closed (reduced) subscheme of $X$.

$w(X):=$ the set of connected components of $\tilde{X} \times{ }_{S} \bar{S}$

$=$ the set of irreducible components of $\bar{X}$.

A variety is a scheme $V$ over $S$ such that $\bar{V}$ is a integral scheme.

We use $S$ and $k$ interchangeably: we say $k$-points of a scheme to mean its points with values in $S$.

The group schemes we consider are commutative. For such a group scheme $G$, let $G^{0}$ denote the connected component of the identity and $G^{0, \text { red }}$ denote the connected component of the identity of the reduced scheme $G^{\text {red }}$. For a positive integer $r$ prime to $p$, we denote by ${ }_{r} G$ the group scheme corresponding to $\operatorname{Ker}(G \stackrel{r}{\rightarrow} G)$, i.e., the $r$-torsion subgroup scheme of $G$.

A semiabelian scheme is a commutative group scheme which is an extension of an abelian scheme by a torus.

$\underline{\mathbb{Z}}$ is the locally algebraic group scheme defined by $\underline{\mathbb{Z}}(\bar{S})=\mathbb{Z}$ with trivial $\mathbf{g}$ action.

$\mathbb{G}^{\prime}:=$ the category of group schemes over $S$.

$\mathbb{G}:=$ the category of locally algebraic semiabelian schemes over $S$.

$\mathbb{D}:=$ the abelian category of sheaves (of abelian groups) on $S_{F L}$.

$S_{F L}:=$ the (big) flat site of $S[26]$.

Properties of a simplicial scheme $X_{\bullet}$ are properties that hold for each $X_{i}$ : $X_{\bullet}$ is proper if each $X_{i}$ is proper over $S$, same for smooth, normal, reduced, etc.

Let $W$ be a set, and denote by $\mathbb{Z}[W]$ the free abelian group generated by $W$. We define the abelian group $\mathbb{Z}^{W}$ to be the group of integer valued functions on $W$ i.e. $\mathbb{Z}^{W}:=\operatorname{Maps}(W, \mathbb{Z})$. For a finite set $W$, the groups $\mathbb{Z}(W)$ and $\mathbb{Z}^{W}$ are naturally dual as abelian groups.

For any finitely generated abelian group $G$, we put $G_{t o r}$ to denote the torsion subgroup of $G$. We denote by $G /$ tor the maximal torsion-free quotient of $G$.

$\mathcal{M H \mathcal { S }}:=$ the abelian category of mixed Hodge structures (MHS).

$\mathcal{M H}_{\text {pol }}^{1}:=$ the category of torsion-free MHS $N$ of type

$$
\{(0,0),(-1,0),(0,-1),(-1,-1)\}
$$

such that $G r_{-1}^{W} N$ is polarizable [6] $\S 10.1$.

$\mathfrak{T}(M) \in \mathcal{M H}_{\text {pol }}^{1}$ denotes the Hodge realization (河 10.1) of a 1-motive $M$.

We use $H_{\mathbb{Z}}$ to denote the underlying $\mathbb{Z}$-lattice of the mixed Hodge structure $H$. We use $W_{i} M$ and $W_{i} H$ to indicate the weight filtration on 1-motives and mixed Hodge structures. The Tate Hodge structure $\mathbb{Z}(1)$ corresponds to the lattice $2 \pi i \mathbb{Z} \subset \mathbb{C}$; the Hodge structure $\mathbb{Z}(n)$ (for $n>0$ ) is defined to be $\mathbb{Z}(1)^{\otimes n}$. For $n<0$, we define $\mathbb{Z}(n)$ to be the Hodge structure dual to $\mathbb{Z}(-n)$ i.e we have $\mathbb{Z}(n)=$ $\operatorname{Hom}_{M H S}(\mathbb{Z}(-n), \mathbb{Z})$. The Tate Hodge structure $\mathbb{Z}(n)$ is of weight $-2 n$ of type $(-n,-n)$. For any mixed Hodge structure $H$, the Tate twist $H(n)$ denotes $H \otimes \mathbb{Z}(n)$. For any scheme $V$ over $\mathbb{C}$, we use $H^{*}(V)$ to indicate the mixed Hodge structure $H^{*}(V(\mathbb{C}) ; \mathbb{Z})$. Similar convention for homology applies as well. Given a sheaf $\mathcal{F}$ on $V$, we write $H^{i}(V ; \mathcal{F})$ for $H^{i}(V(\mathbb{C}) ; \mathcal{F})$.

Acknowledgements. It is a pleasure for me to thank S. Lichtenbaum for much encouragement. The results presented here would not have been possible without his guidance; that they appear in published form is due to his insistence. This paper is a greatly revised version of [29]. I would like to thank the mathematics department at Brown, the Alfred P. Sloan foundation, and the National Science 
Foundation (NSF) for their support. Lastly, I heartily thank the referee for the careful reading, enthusiasm, and numerous suggestions thereby improving the exposition.

\section{Albanese Schemes}

This section is based on a succinct suggestion of J.-P. Serre [37]. The goal is the definition of the Albanese scheme of a scheme $X$ over $S$. This is different from the (very interesting) universal regular quotient of 11.

\subsection{Locally algebraic group schemes.}

Definition 1.1. A locally algebraic group scheme is a group scheme locally of finite type over $S$.

A (group) scheme of finite type will sometimes be called an algebraic (group) scheme. A locally algebraic group scheme $G$ such that $G^{0}=S$ is said to be discrete or of dimension zero; it is an étale $S$-scheme. Such a group $G$ is determined (up to isomorphism) by the discrete g-module $G(\bar{S})$.

Recall from [9] pp. 284-6 that there is a discrete locally algebraic group $\pi_{0}(G)$ associated with a locally algebraic group scheme $G$, a scheme-theoretic analog of the group of connected components; it is the universal object for homomorphisms from $G$ to discrete locally algebraic group schemes. One has an exact sequence of locally algebraic group schemes:

$$
0 \rightarrow G^{0} \rightarrow G \stackrel{q_{G}}{\longrightarrow} \pi_{0}(G) \rightarrow 0
$$

where $q_{G}$ is a homomorphism of group schemes.

1.2. Construction. Let $B$ be a commutative group scheme (algebraic) over $S$. Given two $B$-torsors $P_{1}$ and $P_{2}$ (torsor $=$ principal homogeneous space), the $B$-torsor obtained by Baer summation is denoted $P_{1} \vee_{B} P_{2}$. For any $B$-torsor $P$, one can define a new torsor $P^{-1}$ by twisting the action of $B$ by the automorphism corresponding to the inverse $i: B \rightarrow B$. With each $B$-torsor $P$, one can associate a locally algebraic group $B_{P}$ as follows. For $n$ positive, let $P^{\otimes n}$ denote the $B$-torsor $P \vee_{B} \cdots \vee_{B} P$ ( $n$ terms). For $n$ negative, let $P^{\otimes n}$ denote the $B$-torsor $P^{-1} \vee_{B} \cdots \vee_{B} P^{-1}(n$ terms $)$. Let $P^{\otimes 0}$ be the trivial torsor $B$. We define

$$
B_{P}:=\coprod_{n \in \mathbb{Z}} P^{\otimes n}
$$

note the canonical morphism $a_{P}: B_{P} \rightarrow \underline{\mathbb{Z}}$. The natural morphisms

$$
P^{\otimes n} \times_{S} P^{\otimes m} \rightarrow P^{\otimes n} \vee_{B} P^{\otimes m}:=P^{\otimes(m+n)}
$$

endow $B_{P}$ with the structure of a commutative group scheme; $B_{P}$ is an extension of $\underline{\mathbb{Z}}$ by the group scheme $B$. Any scheme $T$ defines a sheaf $\mathcal{T}$ of sets on $S_{F L}: X \mapsto \operatorname{Hom}_{S}(X, T)$. There is a natural inclusion of $\mathcal{P} \hookrightarrow \mathcal{B}_{P}$ of sheaves of sets on $S_{F L}$; in fact, $\mathcal{P}$ is the subsheaf of $\mathcal{B}_{P}$ corresponding to the inverse image under $a_{P}$ of the section $1 \in \underline{\mathbb{Z}}$. 
A semiabelian scheme is an algebraic group scheme which is an extension of an abelian scheme by a torus; it is geometrically connected. We call a locally algebraic group scheme $G$ semiabelian if $G^{0}$ is a semiabelian scheme. If $B$ is a semiabelian scheme and $P$ is a torsor under $B$, then the group scheme $B_{P}$ is a semiabelian locally algebraic group scheme.

Definition 1.2. A large group is a semiabelian locally algebraic group scheme.

We use this terminology because (1) the name is short and (2) a large group is not a scheme of finite type. We denote by $\mathbb{D}$ the abelian category of sheaves (of abelian groups) on the (big) flat site $S_{F L}$ of $S$. The category $\mathbb{G}$ of large groups embeds as a full subcategory (denoted $\mathbb{G}$ ) of $\mathbb{D}$.

1.3. Morphisms to large group schemes. For any set $W$, let $\mathbb{Z}[W]$ be the free abelian group generated by the set $W$. Let $X$ be a scheme. Define $P_{X}$ to be the presheaf of abelian groups on $S_{F L}$ by $T \mapsto \mathbb{Z}[X(T)]$.

Definition 1.3. $\mathcal{Z}_{X}$ is the sheaf on $S_{F L}$ associated with the presheaf $P_{X}$.

The association $X \mapsto \mathcal{Z}_{X}$ determines a covariant functor from the category of schemes over $S$ to $\mathbb{D}$. If we consider $X$ as a sheaf $\mathcal{X}$ of sets, then we have a canonical inclusion $j: \mathcal{X} \hookrightarrow \mathcal{Z}_{X}$. The sheaf $\mathcal{Z}_{X}$ has the following universal property: Given any abelian sheaf $\mathcal{F}$ on $S_{F L}$ and a map $g: \mathcal{X} \rightarrow \mathcal{F}$ of sheaves of sets, there is a unique homomorphism of abelian sheaves $\tilde{g}: \mathcal{Z}_{X} \rightarrow \mathcal{F}$ which factors $g$, i.e., $\tilde{g} j=g$.

It is known that the sheaf $\mathcal{Z}_{X}$ is not representable in any reasonable topology 40]. One is naturally led to consider the universal representable quotient of $\mathcal{Z}_{X}$; we restrict ourselves to the situation where the representing scheme is a large group.

It is well known that there is an étale algebraic $S$-scheme $\pi_{0}(X)$ and a $S$-morphism $q_{X}: X \rightarrow \pi_{0}(X)$ which is universal for morphisms from $X$ into étale $S$-schemes ([9], Prop 6.5, p.154).

Consider the sheaf $\mathcal{Z}_{\pi_{0}(X)}$ on $S_{F L}$ defined by the scheme $\pi_{0}(X)$; as above, $\tilde{q}_{X}$ denotes the induced homomorphism from $\mathcal{Z}_{X} \rightarrow \mathcal{Z}_{\pi_{0}(X)}$. The following lemma is well known (loc. cit).

Lemma 1.4. (a) $\mathcal{Z}_{\pi_{0}(X)}$ is represented by a discrete large group $D_{X}$.

(b) The pair $\left(\mathcal{Z}_{\pi_{0}(X)}, \tilde{q}_{X}\right)$ is universal for morphisms from $\mathcal{Z}_{X}$ to discrete group schemes.

The group $D_{X}$ of a variety $X$ is isomorphic to $\underline{\mathbb{Z}}$.

Definition 1.5. The Albanese sheaf of $X$ is the universal object for morphisms from $\mathcal{Z}_{X}$ to objects in the subcategory $\mathbb{G}$ (i.e. sheaves corresponding to large groups).

We say that $X$ admits a universal morphism if the Albanese sheaf of $X$ exists. 
Grant, for the moment, the existence of the Albanese sheaf of $X$. The sheaf $\mathcal{A}_{X}$ comes equipped with a surjective homomorphism $u_{X}: \mathcal{Z}_{X} \rightarrow \mathcal{A}_{X}$. Let $A_{X}$ be the large group corresponding to the sheaf $\mathcal{A}_{X}$. The morphism $\mathcal{X} \hookrightarrow \mathcal{Z}_{X} \rightarrow \mathcal{A}_{X}$ of sheafs of sets on $S_{F L}$ corresponds, by Yoneda's lemma, to a morphism $u_{X}: X \rightarrow A_{X}$ of schemes.

We call $A_{X}$ the Albanese scheme of $X$ and $u_{X}$ is the universal morphism associated with $X$. The pair $\left(A_{X}, u_{X}\right)$ is uniquely determined (up to unique isomorphism). An easy consequence of definition 1.5 is a canonical isomorphism

$$
\pi_{0}\left(A_{X}\right) \stackrel{\sim}{\rightarrow} D_{X}
$$

The assignment $X \mapsto A_{X}$ determines a covariant functor $A$ from the category of algebraic schemes over $S$ to large groups over $S$.

For a scheme $W$ finite over $S$ (e.g., a finite group scheme), we have canonical isomorphisms

$$
\mathcal{Z}_{W} \stackrel{\sim}{\rightarrow} A_{W} \stackrel{\sim}{\rightarrow} D_{W}
$$

The next proposition follows from the corresponding properties of the functor $P \mapsto \mathcal{Z}_{P}$.

Proposition 1.6. (Properties of the Albanese scheme)

(i) (base change) Let $S^{\prime}$ be the spectrum of a perfect field $k^{\prime}$ containing $k$. One has $A_{X} \times{ }_{S} S^{\prime}=A_{X \times{ }_{S} S^{\prime}}$.

(ii) Let $X:=Y \amalg V$ be the disjoint union of schemes $Y$ and $V$. One has $A_{X}=A_{Y} \times{ }_{S} A_{V}$.

(iii) Let $X \stackrel{f}{\rightarrow} Y$ be a surjective morphism. The induced map $f_{*}: A_{X} \rightarrow$ $A_{Y}$ is a surjection.

Remark 1.7. Serre has shown that every scheme $X$ admits a universal morphism to a torsor $P$ under a semiabelian scheme $B$ : in [34], he shows that every scheme $X$ admits a universal morphism (over $\bar{k}$ ) to a semiabelian scheme $B$ (the scheme $B$ is defined over $k$ ) and the method of descent as in [36] (pp.102-107) assure the existence of a universal map (defined over $k$ ) to a torsor $P$ under the universal semiabelian scheme $B$. This semiabelian scheme $B$ is the "generalized Albanese" of $X$.

Remark 1.8. Let $T$ (resp. $R$ ) be a torsor under a group scheme $G$ (resp. under $H$ ). Consider the group schemes $G_{T}$ and $H_{R}$ together with the canonical surjective homomorphisms $a_{T}: G_{T} \rightarrow \underline{\mathbb{Z}}$ and $a_{R}: H_{R} \rightarrow \underline{\mathbb{Z}}$. Specifying a morphism $h$ of torsors between $T$ and $R$ is equivalent to specifying a homomorphism of group schemes $\tilde{h}: G_{T} \rightarrow H_{R}$ such that $a_{R} \tilde{h}=a_{T}$ (i.e. $\tilde{h}$ induces the identity on the quotient $\underline{\mathbb{Z}}$ ).

Let $V$ be a connected scheme (so that there is a map $g_{V}: D_{V} \rightarrow \underline{\mathbb{Z}}$ ). Let $f: V \rightarrow P$ be the universal morphism from $V$ to torsors under semiabelian schemes (as in Remark 1.7). Consider the natural inclusion of $P$ in $B_{P}$. 
The map $f$ induces a map $\tilde{f}: \mathcal{Z}_{V} \rightarrow B_{P}$. Define $B^{\prime \prime}:=B_{P} \times_{\underline{\mathbb{Z}}} D_{V}$, where the fiber product is taken via the maps $a_{P}: B_{P} \rightarrow \underline{\mathbb{Z}}$ and $g_{V}$. The map $\tilde{f}$ factorizes as $\mathcal{Z}_{V} \stackrel{f^{\prime \prime}}{\longrightarrow} B^{\prime \prime} \rightarrow B_{P}$ by the universal property of $D_{V}$. The main difference between $B^{\prime \prime}$ and $B_{P}$ lies in their group of connected components.

Proposition 1.9. The pair $\left(B^{\prime \prime}, f^{\prime \prime}\right)$ is the Albanese scheme of $V$.

Proof. Consider a homomorphism $d^{\prime}: \mathcal{Z}_{V} \rightarrow H^{\prime}$ to a large group $H^{\prime}$. By universality of $D_{V}$, the map $d^{\prime}$ factorizes uniquely as $j d$ where

(a) $d: \mathcal{Z}_{V} \rightarrow H$ is a homomorphism to a large group $H$ inducing an isomorphism $D_{V} \stackrel{\sim}{\rightarrow} \pi_{0}(H)$; notice $H=H^{\prime} \times_{\pi_{0}\left(H^{\prime}\right)} D_{V}$.

and

(b) $j: H \rightarrow H^{\prime}$ is a homomorphism of large groups.

It suffices to show that the map $d$ factorizes via $\left(B^{\prime \prime}, f^{\prime \prime}\right)$. Let $\mathcal{H}$ denote the sheaf of abelian groups on $S_{F L}$ corresponding to the group scheme $H$. The composed map $d_{1}: \mathcal{V} \hookrightarrow \mathcal{Z}_{V} \stackrel{d}{\rightarrow} \mathcal{H}$ of sheaves of sets on $S_{F L}$ gives, by Yoneda's lemma, a $S$-morphism $d_{1}: V \rightarrow H$ of schemes. Since $V$ is connected, its image under $d_{1}$ is contained in a connected component $Q$ of $H$. The neutral component $C$ of $H$ is a semiabelian scheme. The scheme $Q$ has the structure of a torsor under $C$ such that the pair of inclusions $Q \hookrightarrow H$ and $C \hookrightarrow H$ combine into a morphism of torsors. In other words, there is a (injective) morphism of group schemes $k: C_{Q} \rightarrow H$. Now we use the fact that $f$ is universal for morphisms from $V$ to torsors under semiabelian schemes. We get a homomorphism $b: B \rightarrow C$ and a morphism of torsors $h: P \rightarrow Q$ such that $d_{1}=h f$. By Remark 1.8, this is equivalent to specifying a homomorphism $\tilde{h}: B_{P} \rightarrow C_{Q}$ compatible with the augmentation to $\underline{\mathbb{Z}}$. We have shown that $d$ can be factorized as $k \tilde{h} \tilde{f}$. But, as we saw, $\tilde{f}$ can be factorized via $f^{\prime \prime}$. This demonstrates the universal property of the pair $\left(B^{\prime \prime}, f^{\prime \prime}\right)$.

Corollary 1.10. Let $A_{V}$ be the Albanese scheme of a connected scheme $V$, as above. The neutral component of $A_{V}$ is the "generalized Albanese variety" of $V$ defined by Serre (1.7).

Theorem 1.11. Let $X$ be a reduced algebraic scheme over $S$. There exists a large group $A_{X}$ together with a map $u_{X}: \mathcal{Z}_{X} \rightarrow \mathcal{A}_{X}$ which is universal for homomorphisms from $\mathcal{Z}_{X}$ to large groups.

Proof. We have just seen that the Albanese scheme exists for connected schemes. Now use Proposition 1.6.

The identification of Corollary 1.10 yields the

Corollary 1.12. (i) Given a torsor $P$ under a semiabelian scheme $B$, the natural inclusion of $P \stackrel{j}{\rightarrow} B_{P}$ induces a homomorphism $\tilde{j}: \mathcal{Z}_{P} \rightarrow B_{P}$. The large group $B_{P}$, together with the map $\tilde{j}$, is the Albanese scheme of $P$. 
(ii) If $X$ is proper, then $A_{X}^{0}$ is an abelian scheme.

(iii) If $U$ is an open dense subscheme of $X$, then the induced map $A_{U} \rightarrow$ $A_{X}$ is surjective.

(iv) (homotopy invariance) As usual, $\mathbb{A}^{n}$ denotes $n$-dimensional affine space over $S$. Let $Y:=X \times{ }_{S} \mathbb{A}^{n}$. The natural projection $\pi: Y \rightarrow X$ induces an isomorphism $\pi_{*}: A_{Y} \stackrel{\sim}{\rightarrow} A_{X}$.

(v) For any semiabelian scheme $B$, one has $A_{B}=B \times_{S} \underline{\mathbb{Z}}$.

(vi) (Kunneth) Let $X:=Y \times_{S} V$ and let $\alpha: D_{X} \rightarrow D_{Y} \times_{S} D_{V}$ denote the natural map. One has

$$
A_{X}=\left(A_{Y} \times{ }_{S} A_{V}\right) \times\left(D_{Y} \times D_{V}\right) D_{X} .
$$

Proof. We prove (iv), (v), (vi) and leave the rest to the reader. For (iv), observe that $A_{\mathbb{A}^{n}}=\underline{\mathbb{Z}}$ : there are no nonconstant morphisms from $\mathbb{A}^{n}$ to semiabelian varieties. Statement (v) is a special case of (i) which follows from Proposition 1.9. As for (vi), it is clear that $\pi_{0}\left(A_{X}\right)=D_{X}$. Now, use the fact that $A_{X}^{0}=A_{Y}^{0} \times_{S} A_{V}^{0}$ which follows (using Corollary 1.10) from the corresponding property for the "generalized Albanese varieties" of Serre (1.7) proved in [34.

Example 1.13. A curve is a scheme of pure dimension one over $S$. Let $X$ be a normal integral curve. There is a unique normal projective curve $X^{\prime}$ containing $X$ as an open dense subscheme; let $R$ be the closed complement of $X$ in $X^{\prime}$. The semiabelian variety $A_{X}^{0}$ is the generalized Jacobian of Rosenlicht [32] of $X^{\prime}$ corresponding to the modulus $R$ (cf. 6.4 and [35] p. $4)$.

\section{SPeCial SCHEMES}

Let $X$ be the open complement of a divisor $E$ in a smooth and projective variety $X^{\prime}$ of dimension $d$. Let $j: X \hookrightarrow X^{\prime}$ denote the inclusion. Schemes such as $X$ are termed special. We fix these notations for the rest of this section. We now turn to a discussion of the "generalized Albanese variety" of $X$ defined by Serre 350.

Let $w(E)$ denote the set of geometric points of $\pi_{0}(\tilde{E})$ i.e. $w(E)$ is the set of connected components of $\tilde{E} \times_{S} \bar{S}$ or, what is the same, the set of irreducible components of $\bar{E}$. Given a set $W$, one defines the abelian group $\mathbb{Z}^{W}$ to be the group of integer valued functions on $W$ i.e. $\mathbb{Z}^{W}:=\operatorname{Maps}(W, \mathbb{Z})$. For a finite set $W$, the groups $\mathbb{Z}(W)$ and $\mathbb{Z}^{W}$ are naturally dual as abelian groups.

The étale group scheme $\underline{\mathbb{Z}}^{E}$ is defined by its geometric points: $\underline{\mathbb{Z}}^{E}(\bar{S})=$ $\mathbb{Z}^{w(E)}$. If $\operatorname{Pic}\left(\bar{X}^{\prime}\right):=H^{1}\left(\bar{X}^{\prime} ; \mathcal{O}_{\bar{X}^{\prime}}^{*}\right)$ denotes the absolute Picard group of $\bar{X}^{\prime}$, there is a natural g-equivariant homomorphism

$$
\mathbb{Z}^{w(E)} \stackrel{b}{\rightarrow} \operatorname{Pic}\left(\bar{X}^{\prime}\right) \quad d \mapsto \mathcal{O}(d) ;
$$


this provides a morphism?:

$$
b: \underline{\mathbb{Z}}^{w(E)} \rightarrow D_{P i c_{X^{\prime}}^{r e d}}
$$

whose kernel is denoted $B$; set $I=B(\bar{S})$. Under the natural duality between $\mathbb{Z}^{w(E)}$ and $\mathbb{Z}(w(E))$, the group $I$ is dual to the subgroup $J$ of divisors of $X^{\prime} \times_{S} \bar{S}$, supported on $E \times_{S} \bar{S}$, which are algebraically equivalent to zero. Consequently, $I$ and $J$ have the same rank. The Néron-Severi group $N S\left(\bar{X}^{\prime}\right)$ of $\bar{X}^{\prime}$ is the group of geometric points of $D_{P_{i c_{X^{\prime}}}}$. There is a natural morphism $B \stackrel{v}{\rightarrow} P i c_{X^{\prime}}^{0}$.

The abelian scheme $A_{X^{\prime}}^{0}$ is the "classical" Albanese variety of $X^{\prime}$. It is the maximal abelian quotient of the semiabelian scheme $A_{X}^{0}$ (the "generalized Albanese variety" of Serre).

2.1. Serre's Construction. Let us review the construction of $A_{X}^{0}$, via the Picard scheme of $X^{\prime}$, due to Serre [35]. Denote by $v$ the natural map from $B$ to the neutral component $P i c_{X^{\prime}}^{0}$ of $P i c_{X^{\prime}}$. This defines the Picard 1-motive of $X$

$$
M^{1}(X):=\left[B \stackrel{v}{\rightarrow} P i c_{X^{\prime}}^{0, r e d}\right] .
$$

The Cartier dual of this 1-motive is the Albanese 1-motive of $X$

$$
M_{1}(X):=[0 \rightarrow G] ;
$$

the group $G$ is an extension of $A_{X^{\prime}}^{0}$ by the torus $T:=\operatorname{Hom}\left(B, \mathbb{G}_{m}\right)$.

Theorem 2.1. (J.-P. Serre) The semiabelian scheme $G$ is naturally isomorphic to $A_{X}^{0}$ [35.

Corollary 2.2. (a) The kernel of the natural morphism $A_{X}^{0} \rightarrow A_{X^{\prime}}^{0}$ is the torus $T=\operatorname{Hom}\left(B, \mathbb{G}_{m}\right)$.

(b) The dimension of the torus $T$ is the rank of the abelian group $I$.

(c) the 1-motives (8) and (9) are independent of the compactification $X^{\prime}$ of $X$.

Let $u: X^{\prime} \rightarrow L^{\prime}$ be a universal morphism (see Remark 1.7) where $L^{\prime}$ is a torsor for $A_{X^{\prime}}^{0}$. One has the classical isomorphisms

$$
\begin{array}{r}
P i c_{A_{X^{\prime}}^{0}}^{0} \cong P i c_{L^{\prime}}^{0} \cong P i c_{X^{\prime}}^{0}, \\
P:=P i c_{X^{\prime}}^{0, r e d} \quad P i c_{P}^{0, r e d} \cong A_{X^{\prime}}^{0} .
\end{array}
$$

Using this, one interprets the map $v$ as specifying certain $\mathbb{G}_{m}$-torsors on $A_{X^{\prime}}^{0}$ (and on $L^{\prime}$ ). The group scheme $G$ is the geometric scheme corresponding to the total space of the $T$-bundle (given by $v$ ) over $A_{X^{\prime}}^{0}$. Likewise, $L$ is the scheme over $L^{\prime}$ corresponding to the total space of the $T$-bundle. Using the universal map $u$, one may pull this back to $X^{\prime}$ to get a $T$-bundle over $X^{\prime}$ with the following property: given any element $b \in \operatorname{Hom}\left(T, \mathbb{G}_{m}\right)=B$, the

\footnotetext{
${ }^{2} \mathrm{Pic}_{X^{\prime}}$ is the Picard scheme of $X^{\prime}$ (see $\$$ )
} 
$\mathbb{G}_{m}$-torsor over $X^{\prime}$ obtained via $b$ corresponds to the line bundle defined by $b$, viewed as a Cartier divisor on $X^{\prime}$. Observe the trivial fact that $X$ is disjoint from the support (contained in $E$ ) of all the elements of $B(\bar{S})$ viewed as Cartier divisors on $X^{\prime}$. Therefore, given any $b$ as above, the $\mathbb{G}_{m}$-torsor defined by $b$ has a canonical trivialization over $X$ : one has a rational section of the $\mathbb{G}_{m}$-torsor whose divisor is exactly $b$, and this is unique up to an element of $H^{0}\left(X^{\prime} ; \mathcal{O}_{X^{\prime}}^{*}\right)$. This section can be used for the trivialization over $X$. Further, this can be done in an uniform manner to derive a morphism $X \rightarrow L$, which is unique up to translations by elements of $T$. Serre [35] shows that this morphism is universal for maps to torsors under semiabelian schemes. This extends, by universality of the sheaf $\mathcal{Z}_{X}$, to a morphism $\mathcal{Z}_{X} \rightarrow G_{L}$ where $G_{L}$ is the large group scheme defined by the $G$-torsor $L$ (as in 1.2). One deduces that this last morphism is the universal morphism for $X$.

The next theorem is true by definition of $M_{1}(X)$ and $M^{1}(X)$. But the proof of the corresponding result for simplicial smooth schemes (proved in §司) is nontrivial.

Theorem 2.3. The 1-motives $M_{1}(X)$ and $M^{1}(X)$ are dual.

Let $Y$ be an open subscheme of $X$ whose complement in $X$ has codimension greater than one. Let $B_{Y}$ be the group dual to the group of divisors of $X^{\prime} \times_{S} \bar{S}$ (supported on $\left(X^{\prime}-Y\right) \times_{S} \bar{S}$ ) which are algebraically equivalent to zero.

Corollary 2.4. The inclusion of $Y$ in $X$ induces an isomorphism of the Albanese schemes $A_{Y} \stackrel{\sim}{\rightarrow} A_{X}$.

Proof. Observe that $B$ and $B_{Y}$ are all the same.

2.2. Hodge realization. Just as a harbinger of later results, we prove a result about the Hodge realization of $M_{1}(X)$. Consider an imbedding $\iota: k \hookrightarrow \mathbb{C}$. By base-change via $\iota$, we may and do assume (in this subsection) that all our schemes are over $\mathbb{C}$. Since we are over an algebraically closed field, there is no essential difference between a group scheme and its torsors. Let $u: X \rightarrow A_{X}^{0}$ be the universal morphism (uniquely determined by the choice of a base point). Recall that $d$ is the dimension of $X$.

Consider the long exact sequence in cohomology (with coefficients in $\mathbb{Z}$ ) of the pair $\left(X^{\prime}, E\right)$ :

$\rightarrow H^{2 d-2}\left(X^{\prime}\right)(d) \stackrel{\alpha}{\rightarrow} H^{2 d-2}(E)(d) \rightarrow H^{2 d-1}\left(X^{\prime}, E\right)(d) \rightarrow H^{2 d-1}\left(X^{\prime}\right)(d) \rightarrow 0$

Poincaré duality gives $H^{2 d-i}\left(X^{\prime}\right)(d) \cong H_{i}\left(X^{\prime}\right)$. By Lefschetz duality, we have that $H^{2 d-i}\left(X^{\prime}, E\right)(d) \cong H_{i}(X)$. The image of $\alpha$ is the dual MHS of the kernel of $\left(H^{0}(E)(-1) \stackrel{\beta}{\rightarrow} H^{2}\left(X^{\prime}\right)\right)$ where $\beta$ sends an element of $H^{0}(E)(-1)$ to its Poincaré dual, an element of $H^{2}\left(X^{\prime}\right)$. The group $H^{0}(E)$ is the free

\footnotetext{
${ }^{3}$ The variety $X$ is always defined over a finitely generated extension of $\mathbb{Q}$. So, we may assume that $k$ is finitely generated over $\mathbb{Q}$.
} 
abelian group $\mathbb{Z}^{w(E)}$ i.e. the group dual to $\mathbb{Z}(w(E))$. The map $b$ in (可) induces a map on the geometric points and there is a natural inclusion of $D_{P i x_{X^{\prime}}}(\mathbb{C})$ in $H^{2}\left(X^{\prime}\right)$, via the exponential sequence. The composed map is the map $\beta$. The kernel of $\beta$ corresponds, under the duality of $\mathbb{Z}^{w(E)}$ and $\mathbb{Z}(w(E))$, to the subgroup $J$ of divisors of $X^{\prime}$, supported on $E$, which are homologically equivalent to zeroft. Hence we find that $\operatorname{Ker} \beta=I$. If $I^{*}=\operatorname{Hom}_{\mathbb{Z}}(I, \mathbb{Z})$, then $\operatorname{Im} \alpha \cong \operatorname{Hom}_{\mathbb{Z}}(I(-1), \mathbb{Z})=I^{*}(1)$; both are MHS of type $(-1,-1)$. So we obtain the exact sequence

$$
0 \rightarrow I^{*}(1) \rightarrow H_{1}(X) \rightarrow H_{1}\left(X^{\prime}\right) \rightarrow 0 .
$$

Theorem 2.5. (Hodge realization)

(i) The map

$$
u_{*}: H_{1}(X ; \mathbb{Z}) / \text { tor } \rightarrow H_{1}\left(A_{X}^{0} ; \mathbb{Z}\right)=: \mathfrak{T}\left(M_{1}(X)\right)
$$

of mixed Hodge structures is an isomorphism.

(ii) There is a natural isomorphism

$$
\mathfrak{T}\left(M^{1}(X)\right) \stackrel{u^{*}}{\rightarrow} H^{1}(X ; \mathbb{Z}(1))
$$

of mixed Hodge structures.

Proof. Recall from (36] Chap. VI, $\S 14$, Prop. 13, p.128) that $u_{*}$ is surjective; universal morphisms are maximal in the sense of loc. cit.

Consider the following commutative diagram:

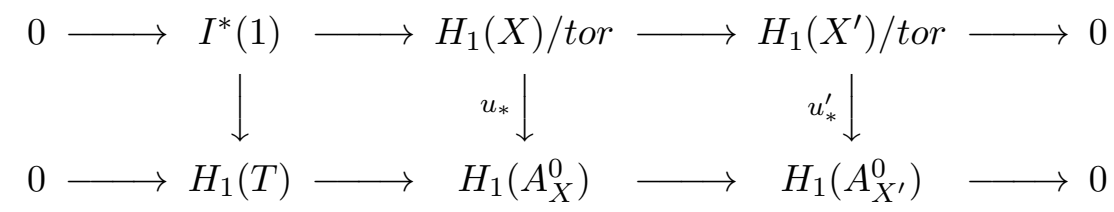

All the vertical maps are maps of MHS because they are induced by morphisms. It is a classical result (see p. 553 of [15) that $u_{*}^{\prime}: H_{1}\left(X^{\prime}\right) /$ tor $\rightarrow$ $H_{1}\left(A_{X^{\prime}}^{0}\right)$ is an isomorphism. By the snake lemma, $I^{*}(1) \rightarrow H_{1}(T)$ is surjective. But the rank of $H_{1}(T)$ is the same as the dimension of $T$ which equals the rank of $I$, as remarked earlier. Since they both have the same rank, the left vertical map is an isomorphism. Thus, we obtain that the map $u_{*}$ : $H_{1}(X) /$ tor $\rightarrow H_{1}\left(A_{X}^{0}\right)$ is a isomorphism of MHS. By definition, $\mathfrak{T}\left(M_{1}(X)\right)$ is the mixed Hodge structure $H_{1}\left(A_{X}^{0} ; \mathbb{Z}\right)$ [6], 10.1.3 p. 54. We turn to (ii). The universal coefficient theorem says that the groups $H_{1}(X ; \mathbb{Z}) /$ tor and $H^{1}(X ; \mathbb{Z})$ are naturally dual. So $H^{1}(X)(1)$ and $H_{1}(X) /$ tor are dual as elements of $\mathcal{M H}_{\text {pol }}^{1}$, i.e., $H^{1}(X)(1)=\operatorname{Hom}_{M H S}\left(H_{1}(X), \mathbb{Z}(1)\right)$. Since duality of 1-motives is compatible with duality in $\mathcal{M H}_{\text {pol }}^{1}$ ([6], §10.2), the result follows from the first part.

\footnotetext{
${ }^{4}$ For divisors, homological equivalence coincides with algebraic equivalence.
} 
Remark 2.6. One cannot expect the previous result to be true for an arbitrary complex algebraic variety (thereby justifying the entrance of 1-motives in this context): the weights in the $H_{1}$ of a semiabelian variety are -2 and -1 , whereas the weights that can and do occur in the $H_{1}$ of an arbitrary variety are $-2,-1$ and 0 ([7] 7.1, 8.3). A noncompact nonrational curve with nodes provides an easy example of the last property.

\section{PiCARD SCHEMES}

In this section, we extend the classical construction of the Picard scheme to a simplicial setting. The arguments are based on spectral sequences.

Let $f: V \rightarrow S$ be a proper reduced scheme of finite type over $S$.

We define $\mathcal{R}^{1} f_{*} \mathcal{O}_{V}^{*}$ on $S_{F L}$ to be the sheaf associated with the presheaf $T \mapsto H^{1}\left(V \times{ }_{S} T ; \mathcal{O}_{V \times{ }_{S} T}^{*}\right)$. By a theorem of J. Murre and F. Oort ([3], $\S 8.2$, Theorem 3, p. 211), the sheaf $\mathcal{R}^{1} f_{*} \mathcal{O}_{V}^{*}$ is representable by a locally algebraic group scheme $P i c_{V}$. One refers to $P i c_{V}$ as the Picard scheme of $V$. One obtains that

$$
H^{1}\left(\bar{V} ; \mathcal{O}_{\bar{V}}^{*}\right)=\operatorname{Pic}(\bar{S})
$$

We recall the well known 9

Proposition 3.1. (i) (de Rham realization) Let $k$ be of characteristic zero. There is a canonical isomorphism ([24], [25], [6] §10.1)

$$
\mathfrak{T}_{d R}\left(\left[0 \rightarrow P i c_{V}^{0}\right]\right) \cong H_{d R}^{1}(V) .
$$

(ii)(Hodge realization) Let $k=\mathbb{C}$. The exponential sequence and G.A.G.A. furnish a natural isomorphism

$$
H^{1}(V)(1) \stackrel{\sim}{\rightarrow} \mathfrak{T}\left(\left[0 \rightarrow P i c_{V}^{0}\right]\right) .
$$

3.1. The Picard scheme of proper simplicial schemes. Let $Z_{\bullet}$ be a simplicial scheme. The absolute Picard group $\operatorname{Pic}\left(Z_{\bullet}\right)$ of $Z_{\bullet}$ is the group $H_{Z a r}^{1}\left(Z_{\bullet} ; \mathcal{O}_{Z_{\bullet}}^{*}\right)$ where $\mathcal{O}_{Z_{\bullet}}^{*}$ is the sheaf of units of the structure sheaf $\mathcal{O}_{Z}$. of $Z_{\bullet}$. We often use the notation $\mathcal{O}^{*}$ to indicate the sheaf of units of a simplicial scheme (specified by the context).

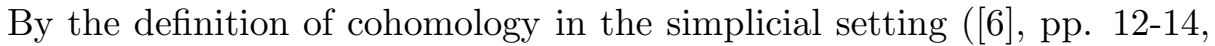
esp. 5.2.3 and 5.2.7, [13], Ex. 1.1, p.7), elements of the group Pic(Z•) correspond to isomorphism classes of pairs $(\mathcal{L}, \alpha)$ where

(1) $\mathcal{L}$ is an invertible sheaf on $Z_{0}$ such that $\delta_{0}^{*} \mathcal{L}$ is isomorphic to $\delta_{1}^{*} \mathcal{L}$;

(2) $\alpha$ is an isomorphism $\delta_{0}^{*} \mathcal{L} \stackrel{\sim}{\rightarrow} \delta_{1}^{*} \mathcal{L}$ on $Z_{1}$ satisfying a cocycle condition on $Z_{2}$, i.e., the following diagram is commutative:

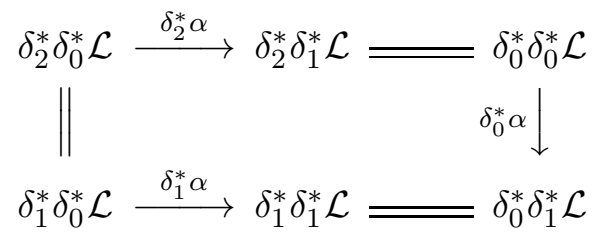

\footnotetext{
${ }^{5}$ The crystalline analog may be found in [18] II 3.11 and [19] pp.46-47.
} 
The maps $\delta_{0}, \delta_{1}: Z_{1} \rightarrow Z_{0}$ and $\delta_{0}, \delta_{1}, \delta_{2}: Z_{2} \rightarrow Z_{1}$ form part of the simplicial structure of the scheme $Z \bullet$ ([6] III, 5.3.6, pp. 15-16). We write the cocycle condition as

$$
\delta_{2}^{*}(\alpha) \delta_{0}^{*}(\alpha)=\delta_{1}^{*}(\alpha)
$$

Let $V_{\bullet}$ be a proper reduced simplicial scheme. The structure morphism of $V_{n} \rightarrow S$ is denoted by $f_{n}$ and the structure map $V_{\bullet} \rightarrow S$ is denoted by $f$. For any scheme $T$ over $S$, we get a simplicial scheme $V_{\bullet} \times_{S} T$. By [6] III, 5.2 .3 .2 , p. 13 , there is a spectral sequence

$$
E_{1, T}^{p, q}=H^{q}\left(V_{p} \times_{S} T ; \mathcal{O}_{V_{p} \times{ }_{S} T}^{*}\right) \Longrightarrow H^{p+q}\left(V_{\bullet} \times{ }_{S} T ; \mathcal{O}^{*}\right) .
$$

We define $\mathcal{R}^{1} f_{*} \mathcal{O}^{*}$ to be the sheaf on $S_{F L}$ associated with the presheaf $E^{1}$ : $T \mapsto H^{1}\left(V_{\bullet} \times_{S} T ; \mathcal{O}^{*}\right)$. The first result that one has about this sheaf is the

Theorem 3.2. The sheaf $\mathcal{R}^{1} f_{*} \mathcal{O}^{*}$ on $S_{F L}$ is representable by a locally algebraic group scheme (denoted $\mathrm{Pic}_{V_{\bullet}}$ ).

Proof. We define $\mathcal{R}^{i} f_{n *} \mathcal{O}_{n}^{*}$ to be the sheaf on $S_{F L}$ associated with the presheaf:

$$
T \mapsto H^{i}\left(V_{n} \times{ }_{S} T ; \mathcal{O}_{V_{n} \times{ }_{S} T}^{*}\right)
$$

The sheaves $\mathcal{R}^{0} f_{n *} \mathcal{O}_{n}^{*}$ are representable by tori. By the classical theorem of Murre and Oort $\left([3], \S 8.2\right.$, Theorem 3, p. 211), the sheaves $\mathcal{R}^{1} f_{n *} \mathcal{O}_{n}^{*}$ are representable by locally algebraic group schemes.

The spectral sequence (13) gives rise to an exact sequence (natural in $T$ ):

$$
0 \rightarrow E_{2, T}^{1,0} \rightarrow H^{1}\left(V_{\bullet} \times{ }_{S} T ; \mathcal{O}^{*}\right) \rightarrow E_{2, T}^{0,1} \rightarrow E_{2, T}^{2,0} .
$$

Let $E^{p, q}$ be the presheaf defined by $T \mapsto E_{2, T}^{p, q}$ and let $\mathcal{E}^{p, q}$ be the sheaf associated with the presheaf $E^{p, q}$.

One has an exact sequence of sheaves on $S_{F L}$ :

$$
0 \rightarrow \mathcal{E}^{1,0} \rightarrow \mathcal{R}^{1} f_{*} \mathcal{O}^{*} \rightarrow \mathcal{E}^{0,1} \stackrel{d_{2}}{\longrightarrow} \mathcal{E}^{2,0} .
$$

The sheaf $\mathcal{E}^{1,0}$ is the homology of the complex of sheaves:

$$
\mathcal{R}^{0} f_{0 *} \mathcal{O}_{0}^{*} \stackrel{\delta_{0}^{*}-\delta_{1}^{*}}{\longrightarrow} \mathcal{R}^{0} f_{1 *} \mathcal{O}_{1}^{*} \stackrel{\delta_{0}^{*}-\delta_{1}^{*}+\delta_{2}^{*}}{\longrightarrow} \mathcal{R}^{0} f_{2 *} \mathcal{O}_{2}^{*}
$$

Each of the sheaves in the above complex is representable by a torus. Therefore the sheaf $\mathcal{E}^{1,0}$ is representable by an affine group scheme $C^{\prime}$ whose neutral component $C$ is a torus.

- The same argument demonstrates the representability of the sheaf $\mathcal{E}^{2,0}$ by an affine group scheme $W$ with $W^{0}$ a torus.

- The sheaf $\mathcal{E}^{0,1}$ is the sheaf $\operatorname{Ker}\left(\mathcal{R}^{1} f_{0 *} \mathcal{O}_{0}^{*} \stackrel{\delta_{0}^{*}-\delta_{1}^{*}}{\longrightarrow} \mathcal{R}^{1} f_{1 *} \mathcal{O}_{1}^{*}\right)$. Since each of these last sheaves is representable by locally algebraic group schemes, we find that the sheaf $\mathcal{E}^{0,1}$ is also representable by a locally algebraic group scheme, denoted $K$. 
- The homomorphism $d_{2}$ corresponds to a morphism of locally algebraic group schemes $K \stackrel{d}{\rightarrow} W$. The kernel of $d$ is a locally algebraic group scheme $Q^{\prime}$ representing the sheaf $\operatorname{Ker}\left(d_{2}\right)$. Thus, in the exact sequence

$$
0 \rightarrow \mathcal{E}^{1,0} \rightarrow \mathcal{R}^{1} f_{*} \mathcal{O}^{*} \rightarrow \operatorname{Ker}\left(d_{2}\right) \rightarrow 0,
$$

the left term is representable by an affine scheme, and the right term is representable. The representability of the sheaf $\mathcal{R}^{1} f_{*} \mathcal{O}^{*}$ follows from the next proposition.

Proposition 3.3. Let $0 \rightarrow F \rightarrow G \rightarrow H \rightarrow$ be an exact sequence of sheaves of abelian groups on $S_{F L}$. Suppose $F, H$ are representable, and that $F \rightarrow S$ is an affine morphism (i.e. the scheme representing $F$ is affine over $S$ ). Then $G$ is representable (necessarily by a commutative group scheme).

Proof. This is Proposition 17.4 on page III.17-6 of [28].

The Picard scheme $P i c_{V_{\bullet}}$ of $V_{\bullet}$ is an extension of $Q^{\prime}$ by the (reduced) affine group scheme $C^{\prime}$. We now recall the ([9] Corollary 2.2, II $\S 5$, no. 2, p. 288):

Theorem 3.4. Let $G$ be a locally algebraic group scheme over a field $F$. If the field $F$ is perfect, then the reduced scheme $G^{\text {red }}$ is a smooth group scheme.

This is the main reason why we assume that $k$ is perfect. Other reasons include

(i) the structure theorems for commutative group schemes are particularly nice over a perfect field (Theorems 1 and 2 of $\S 9.2$ in [3]).

(ii) Over a perfect field, a curve $X$ is smooth if and only if it is normal. These two notions are not equivalent over arbitrary fields (related to the distinction between smoothness and regularity of schemes over imperfect fields; cf. Exercise 10.1 of [17]).

(iii) Grothendieck has expressed doubts about the suitability of 1-motives over imperfect fields (see the appendix to [20]). This is based on the fact that the 1-motives associated with a (singular) curve need not be compatible with base change.

(iv) our eventual interest is in constructing 1-motives for general varieties by using simplicial smooth varieties. Our construction ( $\delta[\overline{]}$ ) is based on the results of [10] which are valid only over perfect fields (page 1 of [10]).

Corollary 3.5. Let $V_{\bullet}$ be a proper reduced simplicial scheme. Assume that $V_{0} \times_{S} \bar{S}$ is normal i.e. $V_{0}$ is geometrically normal. The group scheme Pic $c_{V_{\bullet}}^{0, \text { red }}$ is a semiabelian scheme.

Proof. The sheaf $\mathcal{R}^{1} f_{0 *} \mathcal{O}_{0}^{*}$ is representable by a group scheme whose reduced subscheme is an abelian locally algebraic scheme [16], Theorem 2.1, p. 11. In other words, the neutral component of $K^{\text {red }}$ is an abelian scheme. Recall that every morphism from an abelian variety to an affine group scheme 
is constant; in particular, a homomorphism must necessarily be the zero map. Therefore, the neutral component of $K^{\text {red }}$ must be in the kernel of the morphism $d$. In other words, if $Q$ denotes the neutral component of $\left(Q^{\prime}\right)^{r e d}$, the inclusion of $Q$ in $K^{0, r e d}$ is an equality. We obtain that $\operatorname{Pic}_{V}^{0, \text { red }}$ is a semiabelian scheme.

In particular, Corollary 3.5 applies to a smooth proper simplicial scheme.

Proposition 3.6. Let $V_{\bullet}$ be a proper reduced simplicial scheme. One has the following natural isomorphisms:

(0) $H^{1}\left(V_{\bullet} \times_{S} \bar{S} ; \mathcal{O}^{*}\right) \stackrel{\sim}{\rightarrow} \operatorname{Pic}_{V_{\bullet}}(\bar{S})$

(1) $H^{1}\left(V_{\bullet} ; \mathcal{O}\right) \stackrel{\sim}{\rightarrow}$ Lie Pic $V_{V_{\bullet}}^{0}=$ Lie Pic $V_{\bullet}$

(2) $(k=\mathbb{C}) H^{1}\left(V_{\bullet} ; \mathcal{O}^{*}\right) \stackrel{\sim}{\rightarrow} H^{1}\left(V_{\bullet}^{a n} ; \mathcal{O}^{*}\right), \quad H^{1}\left(V_{\bullet} ; \mathcal{O}\right) \stackrel{\sim}{\rightarrow} H^{1}\left(V_{\bullet}^{a n} ; \mathcal{O}\right)$

(3) $(k=\mathbb{C}) H^{1}\left(V_{\bullet} ; \mathbb{Z}(1)\right) \stackrel{\sim}{\rightarrow} H_{1}\left(\right.$ Pic $\left._{V_{\bullet}}^{0} ; \mathbb{Z}\right)$.

Proof. The isomorphisms in (2) is a natural consequence of G.A.G.A. applied to $E_{2, S}^{p, q}$ of (14). Assertion (0) is clear. The proof of (1) follows the proof of Theorem 1, 88.4 on p. 231 in [3]. In the exact sequence (induced by the exponential sequence on $\left.V_{\bullet}\right)$ :

$$
H^{1}\left(V_{\bullet} ; \mathbb{Z}(1)\right) \stackrel{\partial}{\rightarrow} H^{1}\left(V_{\bullet}^{a n} ; \mathcal{O}\right) \stackrel{\exp }{\longrightarrow} H^{1}\left(V_{\bullet}^{a n} ; \mathcal{O}^{*}\right) \rightarrow H^{2}\left(V_{\bullet} ; \mathbb{Z}(1)\right),
$$

the map $\partial$ is injective since $\exp : \mathbb{C} \rightarrow \mathbb{C}^{*}$ is surjective. By parts $(1)$ and $(2)$, this is enough to prove (3).

\section{The Albanese and Picard 1-motives}

Let us fix a simplicial scheme $X_{\bullet}$, the complement of a divisor $E_{\bullet}$ with normal crossings in a smooth projective simplicial scheme $X_{\bullet}^{\prime}$. In this section, we define the Albanese and Picard 1-motives of $X_{\bullet}$. We prove a conjecture of Deligne that the Hodge realization of the Picard 1-motive of $X_{\bullet}($ over $\mathbb{C}$ ) is $H^{1}\left(X_{\bullet} ; \mathbb{Z}(1)\right)$.

4.1. The Picard 1-motive. Let $R_{n}$ be the large group scheme $\mathbb{Z}^{E_{n}}$. Put $R_{n}{ }_{n}:=\operatorname{Ker}\left(b_{n}: R_{n} \rightarrow D_{\text {Pic }_{X_{n}}}\right)$. We define

$$
R^{\prime}:=\operatorname{Ker}\left(R_{0} \stackrel{\delta_{0}^{*}-\delta_{1}^{*}}{\longrightarrow} R_{1}\right) .
$$

Any element $r$ of $R^{\prime}(\bar{S})$ determines an invertible sheaf $\mathcal{O}(r)$ on $X_{0}^{\prime} \times{ }_{S} \bar{S}$. There is a canonical isomorphism $\alpha_{r}: \delta_{0}^{*} \mathcal{O}(r) \stackrel{\sim}{\rightarrow} \delta_{1}^{*} \mathcal{O}(r)$ on $X_{1}^{\prime} \times_{S} \bar{S}$; the isomorphism $\alpha_{r}$ satisfies the cocycle condition

$$
p_{2}^{*}\left(\alpha_{r}\right) p_{0}^{*}\left(\alpha_{r}\right)=p_{1}^{*}\left(\alpha_{r}\right) .
$$

We have a homomorphism

$$
R^{\prime}(\bar{S}) \stackrel{h}{\rightarrow} \operatorname{Pic}_{X_{\bullet}^{\prime}}(\bar{S})=\operatorname{Pic}\left(X_{\bullet} \times_{S} \bar{S}\right) \quad h^{\prime}(m)=\left([\mathcal{O}(r)], \alpha_{r}\right) .
$$


Let $R^{\prime} \stackrel{h^{\prime}}{\rightarrow} P i c_{X^{\prime}}$ be the corresponding homomorphism of group schemes. Composing with the natural morphism Pic $_{X_{\bullet}^{\prime}} \rightarrow N S\left(X_{\bullet}^{\prime}\right):=\pi_{0}\left(\right.$ Pic $\left._{X_{\bullet}^{\prime}}\right)$, one gets a homomorphism

$$
R^{\prime} \stackrel{h^{\prime \prime}}{\rightarrow} N S\left(X_{\bullet}^{\prime}\right)
$$

whose kernel is denoted $R$. Another description of $R$ is

$$
R=\operatorname{Ker}\left(R_{0}{ }_{0} \stackrel{\delta_{0}^{*}-\delta_{1}^{*}}{\longrightarrow} R{ }_{1}\right) .
$$

Recall from Corollary 3.5 that $P:=P i c_{X_{\bullet}^{\prime}}^{0, \text { red }}$ is a semiabelian scheme.

Definition 4.1. The motivic $H^{1}$ (or the Picard 1-motive) of $X_{\bullet}$ is:

$$
M^{1}\left(X_{\bullet}\right):=[R \stackrel{h}{\rightarrow} P] .
$$

4.2. The Albanese 1-motive. We put $A_{i}=A_{X_{i}}$ and $D_{i}=D_{X_{i}}$; note that $D_{i}=D_{A_{i}}$. By virtue of the simplicial structure of $X_{\bullet}$, the groups $A_{i}$, $A_{i}^{0}, D_{i}$ assemble to form simplicial groups $A_{\bullet}, A_{\bullet}^{0}$ and $D_{\bullet}$; they fit into an exact sequence of simplicial group schemes

$$
0 \rightarrow A_{\bullet}^{0} \rightarrow A_{\bullet} \rightarrow D_{\bullet} \rightarrow 0
$$

There is a natural functor (usually termed normalization) 23 which transforms a simplicial commutative group into a homological complex; the simplicial maps $\delta_{i}$ are replaced by the differentials $d:=\Sigma_{i}(-)^{i} \delta_{i}$. Thus, the previous sequence can be interpreted as an exact sequence of complexes of locally algebraic group schemes (concentrated in nonnegative degrees); as such, one gets a long exact sequence of homology. We set

$$
G:=H_{0}\left(A_{\bullet}^{0}\right)=\frac{A_{0}^{0}}{d\left(A_{1}^{0}\right)}, \quad N:=H_{1}\left(D_{\bullet}\right)=\frac{\operatorname{Ker}\left(d: D_{1} \rightarrow D_{0}\right)}{\operatorname{Im}\left(d: D_{2} \rightarrow D_{1}\right)} .
$$

One has the boundary map $\partial: N \rightarrow G$.

Definition 4.2. The Albanese 1-motive (or the motivic $H_{1}$ ) of $X_{\bullet}$ is

$$
M_{1}\left(X_{\bullet}\right):=[N \stackrel{\partial}{\rightarrow} G] .
$$

Strictly speaking, $N$ has to be torsion-free for $M_{1}\left(X_{\bullet}\right)$ to be a 1-motive; but we can still make sense of the dual 1-motive of $M_{1}\left(X_{\bullet}\right)$. So the torsion in $N$ does not affect matters much.

Remark 4.3. (Constant schemes) If $X \bullet$ happens to be a constant simplicial scheme, i.e., if $X_{i}=X_{0}$ for all $i>0$ and each of the simplicial maps is the identity, then $M_{1}\left(X_{\bullet}\right)=M_{1}\left(X_{0}\right)$ where $M_{1}\left(X_{0}\right)$ of the special scheme $X_{0}$ is defined as in (8).

Theorem 4.4. (Deligne's conjecture) $)^{6}$ Let $k=\mathbb{C}$. One has a natural isomorphism

$$
H^{1}\left(X_{\bullet} ; \mathbb{Z}(1)\right) \rightarrow \mathfrak{T}\left(M^{1}\left(X_{\bullet}\right)\right) .
$$

\footnotetext{
${ }^{6}$ This has been proved (independently) by Barbieri-Viale and Srinivas [2]; it was announced in [1].
} 
Proof. As before, we set $P:=P i c_{X^{\prime}}^{0}$; it is a reduced scheme.

The main step is the construction of a homomorphism $\beta$ from $H^{1}\left(X_{\bullet} ; \mathbb{Z}(1)\right)$ to Lie $P$, the Lie algebra of $P$; note that Lie $P$ is $H^{1}\left(X_{\bullet}^{\prime} ; \mathcal{O}_{X_{\bullet}^{\prime}}\right)$. For this, we closely follow Deligne ([6], 10.3.9-10.3.15, p. 71-74); the modifications are minor.

Lemma 4.5. (i) Let $j_{*}^{m} \mathcal{O}_{X}^{*}$ be the subsheaf of meromorphic functions of $j_{*} \mathcal{O}_{X}^{*}$ on $X_{\bullet}^{\prime}$. One has

$$
H^{1}\left(X_{\bullet} ; \mathbb{Z}(1)\right)=\mathbb{H}^{1}\left(X_{\bullet}^{\prime} ;\left[\mathcal{O}_{X^{\prime}} \stackrel{\exp }{\longrightarrow} j_{*}^{m} \mathcal{O}_{X}^{*}\right]\right) .
$$

$$
H^{1}\left(X_{\bullet} ; \mathbb{C}\right)=\mathbb{H}^{1}\left(X_{\bullet}^{\prime} ;\left[\mathcal{O}_{X^{\prime}} \stackrel{d}{\rightarrow} \Omega_{X_{\bullet}^{\prime}}^{1}(\log E)\right]\right) .
$$

(iii) The inclusion of $H^{1}\left(X_{\bullet} ; \mathbb{Z}(1)\right)$ in $H^{1}\left(X_{\bullet} ; \mathbb{C}\right)$ is defined by the morphism of complexes

$$
\begin{array}{ccc}
\mathcal{O}_{X^{\prime}} \stackrel{\exp }{\longrightarrow} & j_{*}^{m} \mathcal{O}_{X}^{*} \\
\| & d f / f \downarrow \\
\mathcal{O}_{X^{\prime}} \stackrel{d}{\longrightarrow} & \Omega_{X^{\prime}}^{1}(\log E) .
\end{array}
$$

Proof. Consider the commutative diagrams

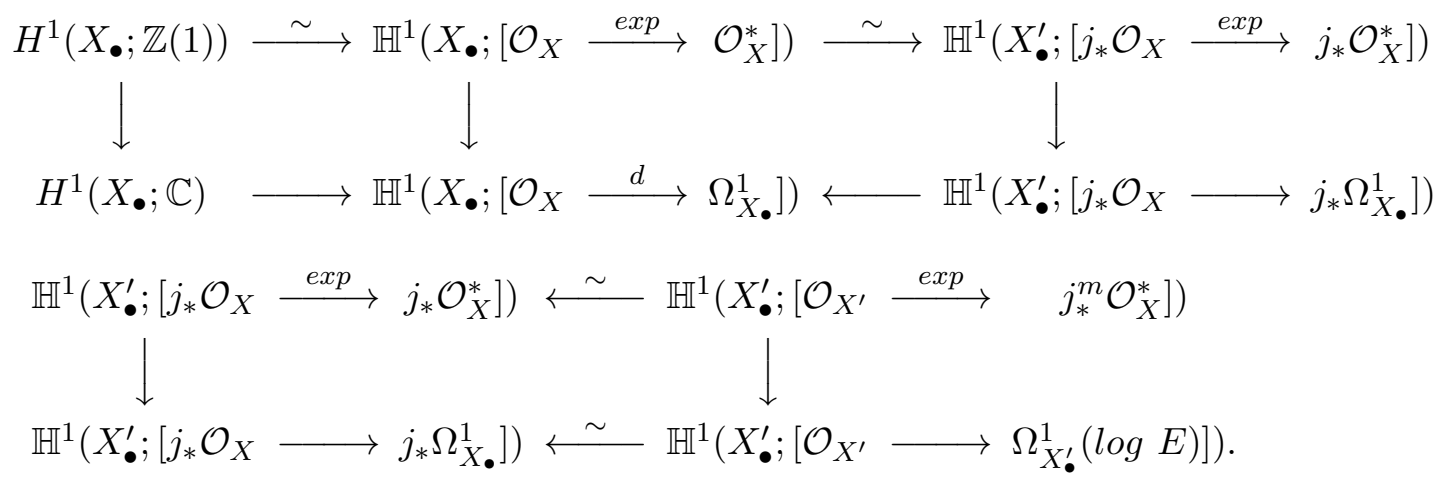

The horizontal maps in the first diagram are isomorphisms due to

(1) the exact sequence of complexes on $X_{\bullet}^{\prime}$ :

$$
0 \rightarrow \mathbb{Z}(1) \rightarrow \mathcal{O}_{X \bullet} \stackrel{\exp }{\longrightarrow} \mathcal{O}_{X \bullet}^{*} \rightarrow 0 .
$$

(2) the fact that the inclusion of complexes

$$
0 \rightarrow \mathbb{C} \rightarrow\left[\mathcal{O}_{X} \stackrel{d}{\rightarrow} \Omega_{X \bullet}^{1}\right]
$$

is a quasi-isomorphism in degree less than 2. More generally, the inclusion of the truncated De Rham complex $\Omega_{V_{\bullet}}^{<n}$ in the De Rham complex of a smooth simplicial scheme $V_{\bullet}$ induces a quasi-isomorphism in degree less than $n$.

(3) the fact that $\mathcal{R}^{1} j_{*} \mathcal{O}_{X \bullet}=0([6]$ 3.1.7).

The horizontal maps in the second diagram are isomorphisms because the morphism of complexes which define them are quasi-isomorphisms. 
The group $\mathbb{H}^{1}\left(X_{\bullet}^{\prime} ;\left[\mathcal{O}_{X_{\bullet}^{\prime}}^{*} \rightarrow \Omega_{X_{\bullet}^{\prime}}^{1}(\log E)\right]\right)$ can be interpreted as the group of isomorphism classes of triples $\left(\mathcal{L}, \alpha_{\mathcal{L}}, \omega_{\mathcal{L}}\right)$, with $\mathcal{L}$ an invertible sheaf on $X_{\bullet}^{\prime}$ (thereby determining $\mathcal{L}_{0}$ on $\left.X_{0}^{\prime}\right), \alpha_{\mathcal{L}}$ is an isomorphism $\delta_{0}^{*}\left(\mathcal{L}_{0}\right) \stackrel{\sim}{\rightarrow} \delta_{1}^{*}\left(\mathcal{L}_{0}\right)$, and $\omega_{\mathcal{L}}$ is an integrable connection on $\mathcal{L}$, regular on $X_{\bullet}$ with at most simple poles along the divisor $E_{\bullet}$ (cf. [25] 2.5, p. 364 or [31], 1.5, p. 47), whereby, using the connections on invertible sheaves $=$ one-forms dictionary, one can identify $\omega_{\mathcal{L}}$ with an element of $H^{0}\left(X_{\bullet}^{\prime} ; \Omega_{X_{\bullet}^{\prime}}^{1}(\log E)\right)$.

If $a$ denotes the projection $X_{\bullet}^{\prime}$ to $S$, then the $S_{F L}$ sheaf $R^{1} a_{*}\left[\mathcal{O}_{X^{\prime}}^{*} \rightarrow\right.$ $\left.\Omega_{X_{\bullet}^{\prime}}^{1}(\log E)\right]$ is representable by a group scheme, denoted $M_{X_{\bullet}}^{\natural}$, whose group of $\mathbb{C}$-points is the group $\mathbb{H}^{1}\left(X_{\bullet}^{\prime} ;\left[\mathcal{O}_{X_{\bullet}^{\prime}}^{*} \rightarrow \Omega_{X_{\bullet}^{\prime}}^{1}(\log E)\right]\right)$. The group scheme $M_{X}^{\natural}$ is an extension of a subgroup (containing $P$ ) of $P i c_{X}$. by the additive group scheme representing the $S_{f p p f}$ sheaf $R^{0} a_{*} \Omega_{X^{\prime}}^{1}(\log E)$. The representability of $R^{1} a_{*}\left[\mathcal{O}_{X_{\bullet}^{\prime}}^{*} \rightarrow \Omega_{X_{\bullet}^{\prime}}^{1}(\log E)\right]$ follows by applying Proposition 3.3 to the exact sequence

$$
0 \rightarrow R^{0} a_{*} \Omega_{X_{\bullet}^{\prime}}^{1}(\log E) \rightarrow R^{1} a_{*}\left[\mathcal{O}_{X_{\bullet}^{\prime}}^{*} \rightarrow \Omega_{X_{\bullet}^{\prime}}^{1}(\log E)\right] \rightarrow R^{1} a_{*} \mathcal{O}_{X_{\bullet}^{\prime}}^{*} ;
$$

details may be found in 30].

Consider an element $r$ of $R(\mathbb{C})$, i.e. a divisor $r_{0}$ supported on $E_{0}$, algebraically equivalent to zero, such that $\delta_{0}^{*}\left(r_{0}\right)=\delta_{1}^{*}\left(r_{0}\right)$ (equality of divisors on $\left.X_{1}^{\prime}\right)$. The invertible sheaf $\mathcal{O}\left(r_{0}\right)$ on $X_{0}^{\prime}$ extends to an invertible sheaf $\mathcal{O}(r)$ on $X_{\bullet}^{\prime}$, equipped with a canonical isomorphism $\alpha_{r}: \delta_{0}^{*}\left(\mathcal{O}\left(r_{0}\right)\right) \cong \delta_{1}^{*}\left(\mathcal{O}\left(r_{0}\right)\right)$, and an integrable connection $\omega_{r}$ on $\mathcal{O}(r)$, regular on $X$ • with atmost simple poles along the divisor $E_{\bullet}$. The connection $\omega_{r}$ or, equivalently the corresponding one-form, may be described as follows: If $f_{i}$ are defining equations for the divisor $r_{0}$ on any open covering $U_{i}$ of $X_{0}^{\prime}$, then $\omega_{r}=d \log \left(f_{i}\right)=d\left(f_{i}\right) / f_{i}$ on $U_{i}$. One defines a map

$$
R \rightarrow M_{X}^{\natural} \quad r \mapsto\left(\mathcal{O}(r), \alpha_{r}, \omega_{r}\right)
$$

The group $H^{1}\left(X_{\bullet} ; \mathbb{C}\right)=\mathbb{H}^{1}\left(X_{\bullet}^{\prime} ;\left[\mathcal{O}_{X_{\bullet}^{\prime}} \rightarrow \Omega_{X_{\bullet}}(\log E)\right]\right)$ is the Lie algebra of $M_{X}^{\natural}$. It can be identified with the isomorphism classes of triples $\left(L, \alpha_{L}, \omega_{L}\right)$, for $L$ an $\mathcal{O}_{X^{\prime}}$-torsor (thereby determining a $\mathcal{O}_{X_{0}^{\prime}}$-torsor $L_{0}$ ), an isomorphism $\alpha_{L}: \delta_{0}^{*}\left(L_{0}\right) \cong \delta_{1}^{*}\left(L_{0}\right)$ and $\omega_{L}$ an integrable connection, as above, on $L$.

The group $H^{1}\left(X_{\bullet} ; \mathbb{Z}(1)\right)=\mathbb{H}^{1}\left(X_{\bullet}^{\prime} ;\left[\mathcal{O}_{X_{\bullet}^{\prime}} \stackrel{\exp }{\longrightarrow} j_{*}^{m} \mathcal{O}_{X_{\bullet}}^{*}\right]\right)$ is the group of isomorphism classes of triples $\left(L, \alpha_{L}, \beta_{L}\right)$, with $L$ an $\mathcal{O}_{X^{\prime}}$-torsor (determining an $\mathcal{O}_{X_{0}^{\prime}}$-torsor $L_{0}$ ), an isomorphism $\alpha_{L}: \delta_{0}^{*}\left(L_{0}\right) \stackrel{\sim}{\rightarrow} \delta_{1}^{*}\left(L_{0}\right)$, and $\beta_{L}$ an isomorphism of the invertible sheaf $\exp (L)$ with an invertible sheaf $\mathcal{O}(r)$ (here $r \in R(\mathbb{C})$ ). The map $\operatorname{Aut}(L)=\mathbb{C} \rightarrow \operatorname{Aut}(\exp (L))=\mathbb{C}^{*}$ is surjective. This allows one to identify $H^{1}\left(X_{\bullet} ; \mathbb{Z}(1)\right)$ with the group of triples $\left(t, \alpha_{t}, r\right)$ for $t$ an isomorphism class of $\mathcal{O}_{X^{\prime}}$-torsor (determining a $\mathcal{O}_{X_{0}^{\prime}}$-torsor $t_{0}$ ) and an isomorphism $\alpha_{t}: \delta_{0}^{*}\left(t_{0}\right) \cong \delta_{1}^{*}\left(t_{0}\right)$, i.e. an element of Lie Pic $X_{X^{\prime}}$, and $r \in R(\mathbb{C})$, defining a divisor concentrated on $E_{\bullet}$ with $\exp (t)$ as image in $P=P i c_{X^{\prime}}^{0}(\mathbb{C})$. In other words, we have defined an isomorphism (of abelian 
groups)

$$
H^{1}\left(X_{\bullet} ; \mathbb{Z}(1)\right) \stackrel{\sim}{\rightarrow} \mathfrak{T}\left(M^{1}\left(X_{\bullet}\right)\right)
$$

The following lemma tells us that this is compatible with the weight filtration and the Hodge filtration.

Lemma 4.6. (i) One has

$$
\begin{array}{r}
W_{1}\left(H^{1}\left(X_{\bullet} ; \mathbb{Z}\right)\right)=\operatorname{Im}\left(\left(H^{1}\left(X_{\bullet}^{\prime} ; \mathbb{Z}\right)\right) \stackrel{j^{*}}{\rightarrow}\left(H^{1}\left(X_{\bullet} ; \mathbb{Z}\right)\right)\right), \\
W_{0}\left(\left(H^{1}\left(X_{\bullet} ; \mathbb{Z}\right)\right)\right)=\operatorname{Im}\left(\operatorname{Ker}\left(H^{1}\left(X_{\bullet}^{\prime} ; \mathbb{Z}\right) \rightarrow H^{1}\left(X_{0} ; \mathbb{Z}\right)\right) \stackrel{j^{*}}{\rightarrow} H^{1}\left(X_{\bullet} ; \mathbb{Z}\right)\right) .
\end{array}
$$

(ii) The spectral sequence defined by the "stupid" filtration of $\left[\mathcal{O}_{X_{\bullet}^{\prime}} \rightarrow \Omega_{X_{\bullet}^{\prime}}^{1}(\log E)\right]$ degenerates and converges to the Hodge filtration on $H^{1}\left(X_{\bullet} ; \mathbb{C}\right)$.

Proof. For the first, let us check that $H^{1}\left(X_{\bullet} \bmod X_{\bullet}^{\prime} ; \mathbb{Z}\right)$ is pure of weight two. This follows from the exact sequence

$$
0 \rightarrow H^{1}\left(X_{\bullet}^{\prime} ; \mathbb{Z}(1)\right) \stackrel{j^{*}}{\rightarrow} H^{1}\left(X_{\bullet} ; \mathbb{Z}(1)\right) \stackrel{\text { Div }}{\longrightarrow} H^{0}\left(E_{\bullet} ; \mathbb{Z}\right) \stackrel{d}{\rightarrow} H^{2}\left(X_{\bullet}^{\prime} ; \mathbb{Z}(1)\right)
$$

(with a step of the Tate twist $\mathbb{Z}(-1)$, since $H^{0}\left(E_{\bullet} ; \mathbb{Z}\right)$ is pure of weight zero), part of the cohomology sequence of the exact sequence of complexes on $X_{\bullet}^{\prime}$

$$
0 \rightarrow\left[\mathcal{O}_{X^{\prime}} \stackrel{\exp }{\longrightarrow} \mathcal{O}_{X^{\prime}}^{*}\right] \rightarrow\left[\mathcal{O}_{X^{\prime}} \stackrel{\exp }{\longrightarrow} j_{*}^{m} \mathcal{O}_{X}^{*}\right] \rightarrow\left[0 \rightarrow \mathbb{Z}\left(E_{\bullet}\right)\right] \rightarrow 0
$$

the last map is the divisor map $j_{*}^{m} \mathcal{O}_{X}^{*} \rightarrow \mathbb{Z}\left(E_{\bullet}\right)$.

The second assertion in (i) follows from examining 8.1.19.1 and 8.1.20 of [6], p.35. As for (ii), it is clear from the definition 3.1.11, 8.1.8, 8.1.12 of [6] of the Hodge filtration.

Back to the proof of Theorem 4.4: Combining the commutativity of the diagram

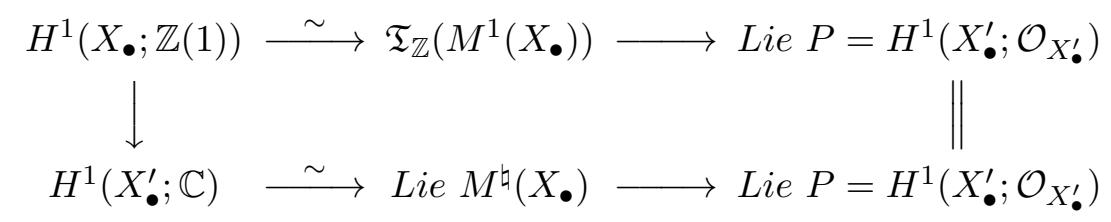

with part (ii) of Lemma 4.6, we see that the map in (16) is compatible with the Hodge filtration.

\section{A Duality theorem}

We retain the notations of $\$$. In this section, we prove the duality of the Albanese and the Picard 1-motives (conjectured by Lichtenbaum). Recall the exact sequence (here $P:=P i c_{X_{\bullet}^{\prime}}^{0, r e d}$ )

$$
0 \rightarrow C^{\prime} \rightarrow P \stackrel{\pi}{\rightarrow} Q \rightarrow 0 .
$$

We set $a: X_{\bullet}^{\prime} \rightarrow S$ to be the natural projection; we use $a_{i}: X_{i}^{\prime} \rightarrow S$ for each of the components.

\footnotetext{
${ }^{7}$ See the definition of $\mathfrak{T}$ [6] $\S 10.1 .3 .1$.
} 


\subsection{The duality theorem.}

Theorem 5.1. (Lichtenbaum's conjecture) The 1-motives

$$
M_{1}\left(X_{\bullet}\right):=[N \stackrel{\partial}{\rightarrow} G], \quad M^{1}\left(X_{\bullet}\right):=[R \stackrel{h}{\rightarrow} P]
$$

are dual.

Remark 5.2. As mentioned earlier, $M_{1}\left(X_{\bullet}\right)$ is not always a 1-motive: $N$ may contain torsion. But it is still possible to define the dual of $M_{1}\left(X_{\bullet}\right)$ (Deligne [6] \$10.2).

Proof. It will be based on a number of lemmas. Let $B$ denote the maximal abelian quotient of $G$; we define the torus $T$ by means of the exact sequence

$$
0 \rightarrow T \rightarrow G \rightarrow B \rightarrow 0 \text {. }
$$

Lemma 5.3. The abelian schemes $B$ and $Q$ are dual.

Proof. The semiabelian scheme $G$ is defined by the top horizontal exact sequence

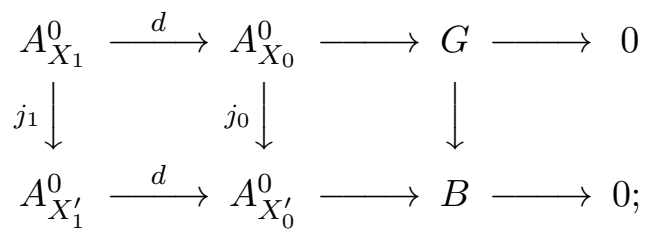

the vertical maps are surjective, taking each semiabelian scheme to its maximal abelian quotient. The abelian scheme $Q$ is the neutral component of the (reduced) kernel of $P i c_{X_{0}^{\prime}}^{0, \text { red }} \stackrel{d_{0}-d_{1}}{\longrightarrow} P i c_{X_{1}^{\prime}}^{0, \text { red }}$. The classical duality of the Albanese and Picard varieties - for any smooth projective scheme $Z$, the abelian schemes $A_{Z}^{0}$ and $P i c_{Z}^{0, \text { red }}$ are naturally dual (compatible with morphisms). Applying this to $X_{0}^{\prime}$ and $X_{1}^{\prime}$, and using functoriality, we see that $Q$ and $B$ are dual.

Lemma 5.4. The torus $\mathcal{H o m}\left(R, \mathbb{G}_{m}\right)$ is isomorphic to $T$.

Proof. In the previous commutative diagram, the torus corresponding to the kernel of $j_{1}$ (resp. $j_{0}$ ) was identified to be $\mathcal{H o m}\left(R{ }_{1}, \mathbb{G}_{m}\right.$ ) (resp. $\left.\mathcal{H o m}\left(R{ }_{0}, \mathbb{G}_{m}\right)\right)$ by Corollary 2.2 (a) applied to $X_{0}$ and $X_{1}$. Consequently $\mathcal{H o m}\left(R, \mathbb{G}_{m}\right)$ can be identified with $T$.

Lemma 5.5. There is a canonical isomorphism of tori:

$$
\mathcal{H o m}\left(N, \mathbb{G}_{m}\right) \cong C^{\prime} .
$$

Proof. Fix a smooth proper scheme $W$ with projection $g: W \rightarrow S$. The torus $\mathcal{H o m}\left(D_{W}, \mathbb{G}_{m}\right)$ also represents the fppf sheaf $R^{0} g_{*} \mathcal{O}_{W}^{*}$; a character of $D_{W}$ provides a nonzero function, constant (since $W$ is proper) on each connected component of $W$, i.e., on each irreducible component since $W$ is 
smooth. The affine group scheme $C^{\prime}$ represents the homology sheaf of the complex :

$$
\mathcal{R}^{0} a_{0 *} \mathcal{O}_{X_{0}^{\prime}}^{*} \stackrel{\delta_{0}^{*}-\delta_{1}^{*}}{\longrightarrow} \mathcal{R}^{0} a_{1 *} \mathcal{O}_{X_{1}^{\prime}}^{*} \stackrel{\delta_{0}^{*}-\delta_{1}^{*}+\delta_{2}^{*}}{\longrightarrow} \mathcal{R}^{0} a_{2 *} \mathcal{O}_{X_{2}^{\prime}}^{*}
$$

this can be identified with the following complex:

$$
\mathcal{H o m}\left(D_{X_{0}^{\prime}}, \mathbb{G}_{m}\right) \stackrel{\delta_{0}^{*}-\delta_{1}^{*}}{\longrightarrow} \mathcal{H o m}\left(D_{X_{1}^{\prime}}, \mathbb{G}_{m}\right) \stackrel{\delta_{0}^{*}-\delta_{1}^{*}+\delta_{2}^{*}}{\longrightarrow} \mathcal{H o m}\left(D_{X_{2}^{\prime}}, \mathbb{G}_{m}\right) .
$$

Thus, $C^{\prime}$ represents the homology sheaf of (18). We have defined $N$ to be the étale group scheme representing the homology of the complex

$$
D_{X_{2}} \stackrel{\delta_{0}-\delta_{1}+\delta_{2}}{\longrightarrow} D_{X_{1}} \stackrel{\delta_{0}-\delta_{1}}{\longrightarrow} D_{X_{0}} .
$$

But note $D_{X_{n}} \cong D_{X_{n}^{\prime}}$ since the complement $E_{\bullet}$ of $X_{\bullet}$ in $X_{\bullet}^{\prime}$ is a simplicial divisor. As the functor $\mathcal{H o m}\left(-, \mathbb{G}_{m}\right)$ establishes an anti-equivalence of the category of group schemes of multiplicative type and étale group schemes, we get that $C^{\prime} \cong \mathcal{H o m}\left(N, \mathbb{G}_{m}\right)$.

A consequence is the isomorphism $\operatorname{Hom}\left(C^{\prime}, \mathbb{G}_{m}\right) \cong N$. In the next lemma, $\pi$ denotes the projection $P \rightarrow Q$ (as above).

Lemma 5.6. The 1-motive $[R \stackrel{\pi h}{\longrightarrow} Q]$ is dual to $[0 \rightarrow G]$.

Proof. As observed earlier, the 1-motive $[R \stackrel{\pi h}{\longrightarrow} Q]$ is the

$$
\operatorname{Ker}\left(\left[R_{0}^{\prime \prime} \rightarrow P i c_{X_{0}^{\prime}}^{0}\right] \stackrel{\delta_{0}^{*}-\delta_{1}^{*}}{\longrightarrow}\left[R_{1}^{\prime \prime} \rightarrow P i c_{X_{1}^{\prime}}^{0}\right]\right),
$$

rendering its dual to be the 1-motive $[0 \rightarrow G]$, by Theorem 2.1 applied to $X_{0}$ and $X_{1}$.

For any 1-motive $M$, Deligne ([6] 10.2.11, p. 67) defines the dual 1-motive $M^{*}$ by $M^{*}:=\mathcal{R} H \operatorname{Hom}\left(M, \mathbb{G}_{m}[-1]\right)$ where $\mathcal{R} H o m$ is the derived functor of $H o m$ on the abelian category of complexes of group schemes over $S$. We follow his prescription in determining the dual of $M:=M_{1}\left(X_{\bullet}\right)$ :

(a) The dual of $M / W_{-2}(M)$

Recall that $P(V)=P i c_{X_{\bullet}^{\prime}}^{0, r e d}(V)$ classifies isomorphism classes of pairs $(\mathcal{L}, \alpha)$ with $\mathcal{L}$ an invertible sheaf on $X_{0}^{\prime} \times{ }_{S} V$ and $\alpha$ an isomorphism $\delta_{0}^{*}(\mathcal{L}) \cong$ $\delta_{1}^{*}(\mathcal{L})$ over $X_{1}^{\prime} \times{ }_{S} V$ satisfying a cocycle relation (12) on $X_{2}^{\prime} \times_{S} V$. We may also interpret $\alpha$ as a trivialization of the invertible sheaf $\delta_{0}^{*}(\mathcal{L}) \otimes\left(\delta_{1}^{*}(\mathcal{L})\right)^{-1}$, i.e., an isomorphism $\delta_{0}^{*}(\mathcal{L}) \otimes\left(\delta_{1}^{*}(\mathcal{L})\right)^{-1} \cong \mathcal{O}_{X_{1} \times S}$ (also required to satisfy a cocycle condition).

There is a natural morphism of functors $\mathcal{E} x t^{1}\left([N \stackrel{\partial}{\rightarrow} B], \mathbb{G}_{m}\right) \stackrel{\psi}{\rightarrow} P$ : elements of the former are isomorphism classes of pairs $(\mathcal{L}, \alpha)$ where $\mathcal{L}$ is an invertible sheaf on $X_{0}^{\prime}$, defined by an element of $Q=\mathcal{E} x t^{1}\left(B, \mathbb{G}_{m}\right)$ (the dual abelian scheme), and $\alpha$ is a trivialization of $\partial^{*}(\mathcal{L})$. The element $\mathcal{L}$ naturally extends to an invertible sheaf on $X_{\bullet}^{\prime}$ because $\mathcal{L} \in Q$. Since $\partial$ is defined via the map $\delta_{0}-\delta_{1}: A_{X_{1}} \rightarrow A_{X_{0}}$, we see that a trivialization of $\partial^{*}(\mathcal{L})$ amounts 
to a trivialization of the invertible sheaf $\delta_{0}^{*}(\mathcal{L}) \otimes\left(\delta_{1}^{*}(\mathcal{L})\right)^{-1}$ on $X_{1}^{\prime}$. Comparing with the definition of $P$ (recalled above), it is clear that the morphism $\psi$ is actually an isomorphism of functors. The 1-motives $[N \stackrel{\partial}{\rightarrow} B]$ and $[0 \rightarrow P]$ are dual.

(b) For each $r \in R \cong \operatorname{Hom}\left(T, \mathbb{G}_{m}\right)$, the extension $M$ of $M / W_{-2}(M)$ by $T$ defines an extension of $M / W_{-2}(M)$ by $\mathbb{G}_{m}$, and hence an element $s(r) \in P$. This combines into a homomorphism $s: R \rightarrow P$. Therefore, the dual of $M_{1}\left(X_{\bullet}\right)$ is the 1 -motive $[R \stackrel{s}{\rightarrow} P]$.

Thus, to finish the proof of Theorem 5.1, it suffices to show that $h=s$.

Let $T_{0}$ be the torus $\operatorname{Hom}\left(R_{0}^{\prime \prime}, \mathbb{G}_{m}\right)$. It is the kernel of the projection $j_{*}: A_{X_{0}} \rightarrow A_{X_{0}^{\prime}}$ (see 2.1). One may view $A_{X_{0}}^{0}$ as the total space of a $T_{0}$-bundle over $A_{X_{0}^{\prime}}^{0}$. Similarly, $L$ (the universal torsor for $X_{0}^{\prime}$ ) is the total space of a $T_{0}$-bundle over $L^{\prime}$. Fix the universal morphisms $u: X_{0} \rightarrow L$, $u^{\prime}: X_{0}^{\prime} \rightarrow L^{\prime}$ and the isomorphisms

$$
P i c_{A_{X_{0}^{\prime}}^{0}}^{0, \text { red }} \cong P i c_{L^{\prime}}^{0, \text { red }} \cong P i c_{X_{0}^{\prime}}^{0, r e d} .
$$

It is a complete tautology that both the $T_{0}$-bundles have the following property: For any $r \in R_{0}^{\prime \prime} \cong \operatorname{Hom}\left(T_{0}, \mathbb{G}_{m}\right)$, the line bundle obtained via $r$ over $A_{X_{0}^{\prime}}^{0}$ and $L^{\prime}$ corresponds to the element $[\mathcal{O}(r)] \in P i c_{X_{0}^{\prime}}^{0, \text { red }}$ under the isomorphisms in (19). Lemma 5.6 and the fact that $R \hookrightarrow R_{0}^{\prime \prime}$ together show that $\pi(s(r))=\pi(h(r))$.

Consider the restriction of the $T_{0}$-bundle $\tilde{L}:=\left(u^{\prime}\right)^{*} L$ over $X_{0}^{\prime}$ to the open subscheme $X_{0}$. The universal morphism $u: X_{0} \rightarrow L$ is defined by lifting the map $u^{\prime}: X_{0} \rightarrow L^{\prime}$ by means of a consistent choice of sections of the $T_{0^{-}}$ bundle $\tilde{L}$. For any $r \in R$, the map $u$ determines a rational section $\tau_{r}$ of $\mathcal{O}(r)$ on $X_{0}^{\prime}$ whose divisor is $r$. On $X_{0}$, the section $\tau_{r}$ of $\mathcal{O}(r)$ is nowhere vanishing and regular. Similarly, we have a rational section $\tau_{-r}$ of the invertible sheaf $\mathcal{O}(-r)$. One has a a priori rational section of the invertible sheaf $\mathcal{L}_{1}:=$ $\delta_{0}^{*}(\mathcal{O}(r)) \otimes \delta_{1}^{*}(\mathcal{O}(r))^{-1}$ on $X_{1}^{\prime}$ given by $\zeta_{r}:=\delta_{0}^{*}\left(\tau_{r}\right) \otimes \delta_{1}^{*}\left(\tau_{-r}\right)$; this is because $\mathcal{O}(r)^{-1}=\mathcal{O}(-r)$. But $\mathcal{L}_{1}$ is the trivial invertible sheaf $\mathcal{O}_{X_{1}}$ on $X_{1}$ and the section $\zeta_{r}$ on $X_{1}$ is the identity section.

The element $s(r)$ corresponds to a pair $\left(\pi(s(r)), \beta_{r}\right)$ where $\beta_{r}$ is a trivialization of the invertible sheaf corresponding to $\pi(s(r))$ over $N$. As remarked earlier, the element $\pi(s(r))$ corresponds to the invertible sheaf $\mathcal{O}(r)$ on $X_{0}^{\prime}$. Utilizing the definition of the map $\partial$, we see that the pull back $\partial^{*}(\mathcal{O}(r))$ is the invertible sheaf $\mathcal{L}_{1}$ on $X_{1}^{\prime}$. Since $\beta_{r}$ is the pullback (via $\partial$ ) of the trivialization $\tau_{r}$ of $\mathcal{O}(r)$ on $X_{0}$, we obtain that $\beta_{r}=\zeta_{r}$ on $X_{1}$. Therefore, $\beta_{r}$ also corresponds to the identity trivialization of $\mathcal{O}_{X_{1}^{\prime}}$. Comparing with the definition of $h$ (recalled below), we obtain that $h(r)=s(r)$.

One could alternatively proceed using the fact that $D_{X_{i}}=D_{X_{i}^{\prime}}$ for any $i$, and the sequences (17) and (18). 
Let us recall the definition of the map $h$ : Any element $r \in R$ satisfies $\delta_{0}^{*}(r)=\delta_{1}^{*}(r)=r_{1}$ (equality of divisors on $X_{1}$ ). Therefore, there is a canonical isomorphism $\alpha_{r}: \delta_{0}^{*}(\mathcal{O}(r)) \cong \delta_{1}^{*}(\mathcal{O}(r))$, corresponding to the identity in $\operatorname{Hom}_{X_{1}^{\prime}}\left(\mathcal{O}\left(r_{1}\right), \mathcal{O}\left(r_{1}\right)\right)$. The invertible sheaf $\delta_{0}^{*}(\mathcal{O}(r)) \otimes\left(\delta_{1}^{*}(\mathcal{O}(r))\right)^{-1}$ is canonically isomorphic to $\mathcal{O}_{X_{1}^{\prime}}$; the element $\alpha_{r}$, interpreted as a trivialization of the former, corresponds to the identity section of $\mathcal{O}_{X_{1}^{\prime}}$. The map $h$ sends $r$ to the pair $\left(\mathcal{O}(r), \alpha_{r}\right)$.

Consequently, the dual 1-motive of $M_{1}\left(X_{\bullet}\right)$ is $M^{1}\left(X_{\bullet}\right)$.

Corollary 5.7. The Picard 1-motive $M^{1}\left(U_{\bullet}\right)$ is a contravariant functor.

Proof. Combine Theorem 5.1 with the (evident) covariant functoriality of the Albanese 1-motives.

Remark 5.8. Theorem 5.1 depends on the following two facts:

(1) For any $r \in R$, the pullback $\delta_{0}^{*}(\mathcal{O}(r)) \otimes \delta_{1}^{*}\left(\mathcal{O}(r)^{-1}\right)$ is the trivial invertible sheaf $\mathcal{O}_{X_{1}^{\prime}}$ on $X_{1}^{\prime}$; this is by definition of the group $R$.

(2) the pullback of any rational section $\tau_{r}$ of $\mathcal{O}(r)$ (such that the divisor of $\tau_{r}$ is precisely $r$ ) under the map $\partial$ is the identity section of $\mathcal{O}_{X_{1}^{\prime}}$; this is by definition of the map $\partial$.

\section{Curves}

A curve denotes a scheme of pure dimension one. For any curve $C$ over an algebraically closed field, there is the motivic $H^{1}$ of $C$ denoted $H_{m}^{1}(C)(1)(1)$ defined by Deligne [6] $\$ 10.3$, and the homological 1-motive $h_{1}(C)$ defined by Lichtenbaum [21]; it is clear by inspection that their definitions are valid over a perfect field $k$.

In this section, we provide a new proof (based on Theorem 5.1) of an unpublished theorem of Lichtenbaum that $h_{1}(C)$ is dual to $H_{m}^{1}(C)(1)$.

We refer to ([3] pp. 247-248; [39]; [22]) for the details of seminormalization. We recall that the seminormalization $\hat{C}$ of a curve $C$ is the largest curve between the normalization $\tilde{C}$ and $C$ which is universally homeomorphic to $C$. The association of $\hat{C}$ and $\tilde{C}$ with $C$ is compatible with base change; this requires $k$ to be perfect.

The 1-motives $H_{m}^{1}$ and $h_{1}$ of curves do not change upon seminormalization and depend only on the underlying reduced scheme structure. Therefore, we may assume that we deal with reduced curves which are seminormal (i.e., that they do not have cuspidal singularities).

6.1. Jacobians of proper curves: a review. Let $L$ be the (reduced) closed subscheme of a seminormal proper curve $C$ corresponding to the singular locus of $C$. Take $g: \tilde{C} \rightarrow C$ to be the canonical projection. Put $K:=L \times_{C} \tilde{C}$; it is a reduced closed subscheme of $\tilde{C}$. There is a natural homomorphism $g: D_{K} \rightarrow D_{L}$ with kernel $D^{\prime}$. The inclusion of $K$ in $\tilde{C}$ provides a homomorphism $D_{K} \rightarrow D_{\tilde{C}}$. This yields a map $D^{\prime} \stackrel{\mu}{\rightarrow} D_{\tilde{C}}$. 
Since $\tilde{C}$ is normal, the connected components of $\tilde{C}$ are the irreducible components of $\tilde{C}$. So one has an isomorphism $\pi_{0}\left(\operatorname{Pic}_{\tilde{C}}\right) \stackrel{\sim}{\rightarrow} \pi_{0}\left(A_{\tilde{C}}\right)=D_{\tilde{C}}$. Using this, one can state the Abel-Jacobi theorem as follows:

Theorem 6.1. (Abel-Jacobi) For any smooth projective curve $X$, one has a natural isomorphism

$$
A_{X} \stackrel{\sim}{\rightarrow} P i c_{X}
$$

this isomorphism is induced by the map $X \hookrightarrow P i c_{X}$ (each point of $X$ is viewed as a divisor).

Consider the exact sequence of sheaves on $C$ :

$$
1 \rightarrow \mathcal{O}_{C}^{*} \rightarrow g_{*} \mathcal{O}_{\tilde{C}}^{*} \rightarrow g_{*} \mathcal{O}_{\tilde{C}}^{*} / \mathcal{O}_{C}^{*} \rightarrow 1 \text {. }
$$

The quotient sheaf $\mathcal{V}:=g_{*} \mathcal{O}_{\tilde{C}}^{*} / \mathcal{O}_{C}^{*}$ is supported on $L$. Let $f: C \rightarrow S$ be the structure morphism. The sequence (20) furnishes an exact sequence of sheaves on $S_{F L}$ :

$$
\begin{aligned}
1 \rightarrow R^{0} f_{*} \mathcal{O}_{C}^{*} \rightarrow R^{0} f_{*}\left(g_{*} \mathcal{O}_{\tilde{C}}^{*}\right) \stackrel{\nu}{\rightarrow} R^{0} f_{*} \mathcal{V} \rightarrow \\
\quad \rightarrow R^{1} f_{*} \mathcal{O}_{\hat{C}}^{*} \rightarrow R^{1} f_{*}\left(g_{*} \mathcal{O}_{\tilde{C}}^{*}\right) \rightarrow 1
\end{aligned}
$$

Since $g$ is finite, its higher derived functors are zero. Thus $R^{1} f_{*}\left(g_{*} \mathcal{O}_{\tilde{C}}^{*}\right)=$ $R^{1}(f g)_{*} \mathcal{O}_{\tilde{C}}^{*}$. In other words, the last sheaf in (21) corresponds to the Picard scheme of $\tilde{C}$.

The sheaf $R^{0} f_{*} \mathcal{V}$ is the torus dual to the group $D^{\prime}$. The sheaf $R^{0} f_{*}\left(g_{*} \mathcal{O}_{\tilde{C}}^{*}\right)$ is the torus dual to $D_{\tilde{C}}$. The map $\nu$ between these two sheaves in corresponds to the pullback of functions by the natural inclusion of $K \hookrightarrow \tilde{C}$. Therefore $\nu$ is dual to the map $D^{\prime} \stackrel{\mu}{\rightarrow} D_{\tilde{C}}$ mentioned above. The cokernel of $\nu$ is the torus $T$ dual to the group scheme $D:=\operatorname{Ker}\left(D^{\prime} \stackrel{\mu}{\rightarrow} D_{\tilde{C}}\right)$.

An immediate corollary of (21) is that the Picard scheme of $C$ also represents the functor which assigns, to a $S$-scheme $Z$, the group of isomorphism classes of pairs $(\mathcal{L}, \alpha)$ where $\mathcal{L}$ is an invertible sheaf on $\tilde{C} \times{ }_{S} Z$ and $\alpha$ is a trivialization of the restriction of $\mathcal{L}$ to $K \times_{S} Z$.

Remark 6.2. Note that the Picard scheme of a proper curve is always reduced [16] Proposition 2.10.

6.2. 1-motives of curves. Let $C$ be a curve (assumed to be seminormal). Denote by $C^{\prime}$ the unique proper curve containing $C$ as a dense open subscheme such that the singular locus of $C^{\prime}$ is the same as that of $C$. As usual, $\tilde{C}$ and $\tilde{C}^{\prime}$ denote their normalizations 5 . We may also describe the latter as the unique smooth proper curve containing $\tilde{C}$ as an open dense subscheme. Denoting by $E$ (resp. $E_{0}$ ) the closed complement of $C(\operatorname{resp} \tilde{C})$ in $C^{\prime}$ (resp. $\tilde{C}^{\prime}$ ), there is a natural projection $c: E_{0} \rightarrow E$, an isomorphism of finite étale schemes. One has an isomorphism

$$
c^{*}: \underline{\mathbb{Z}}^{w(E)} \stackrel{\sim}{\longrightarrow} \underline{\mathbb{Z}}^{w\left(E_{0}\right)} .
$$

\footnotetext{
${ }^{8}$ Note that there is no ambiguity here: $\tilde{C}^{\prime}=(\tilde{C})^{\prime}$
} 
Consider the natural morphism $\alpha: \underline{\mathbb{Z}}^{w(E)} \rightarrow P i c_{C^{\prime}} \rightarrow \pi_{0}\left(P i c_{C^{\prime}}\right)$; let $v$ denote the induced map $\operatorname{Ker}(\alpha) \rightarrow \operatorname{Pic}_{C^{\prime}}^{0}$.

Definition 6.3. (Deligne) The motivic $H^{1}$ of $C$ is the 1-motive

$$
H_{m}^{1}(C)(1):=\left[\operatorname{Ker}(\alpha) \stackrel{v}{\rightarrow} \operatorname{Pic}_{C^{\prime}}^{0}\right] \text {. }
$$

We recall that the special simplicial scheme $\left((\tilde{C} / C)^{\Delta_{n}}\right)_{n \geq 0}$ is smooth (the $(n+1)$-fold fibre product $(\tilde{C} / C)^{\Delta_{n}}$ is the disjoint sum of $\tilde{C}$, the diagonal, and a finite étale scheme) and that it admits $\left(\left(\tilde{C}^{\prime} / C^{\prime}\right)^{\Delta_{n}}\right)_{n \geq 0}$ as a smooth compactification with a (smooth!) simplicial divisor $E_{\bullet}$ as complement. Let us put

$$
C_{n}:=(\tilde{C} / C)^{\Delta_{n}}, \quad C_{n}^{\prime}:=\left(\tilde{C}^{\prime} / C^{\prime}\right)^{\Delta_{n}} \quad(n \geq 0) .
$$

Both of the schemes $C_{\bullet}$ and $C_{\bullet}^{\prime}$ are smooth simplicial schemes. We have $C_{0}=\tilde{C}$ and $C_{0}^{\prime}=\tilde{C}^{\prime}$.

The natural augmentations $a: C_{\bullet} \rightarrow C$ and $b: C_{\bullet}^{\prime} \rightarrow C^{\prime}$ are proper hypercoverings of $C$ and $C^{\prime}$ respectively.

Lemma 6.4. The map $b^{*}: P_{i c_{C^{\prime}}^{0}}^{0} \rightarrow P i c_{C_{\bullet}^{\prime}}^{0}$ is an isomorphism.

Proof. The map from $b_{0}: \tilde{C}^{\prime}=C_{0}^{\prime} \rightarrow C^{\prime}$ is finite and surjective. Interpreting the conditions on the elements of $P i c_{C^{\prime}}^{0}$ as descent data, we see that the map $b^{*}$ is injective $([3], \S 6)$ (see also 6.1).

For the surjectivity, one uses the well known fact that descent data for invertible sheaves is effective for finite surjective morphisms ([3], $\S 6.5$, Theorem 1, p. 157).

Let $F$ denote the singular locus of $C$; we take $F_{0}:=F \times_{C} \tilde{C}$. Denote by $V$ the proper seminormal curve corresponding to the modulus $E_{0}$ on $\tilde{C}^{\prime}$ ([36] IV, no. 4, p. 61 and 76). There is a distinguished $S$-point $U$ which is the unique singular point of $V$. The pullback of $U$ under the map $\tilde{C}^{\prime} \rightarrow V$ is $E_{0}$. The curve $\tilde{C}^{\prime}$ is the normalization of $V$. We set $H:=P i c_{V}^{0}$.

We recall the following

Theorem 6.5. (Rosenlicht-Serre) There is a natural isomorphism

$$
H \stackrel{\sim}{\rightarrow} A_{\tilde{C}}^{0}
$$

of semiabelian schemes over $S$.

Proof. This follows by combining

- Theorem 1 in (36 Chapter V, p.88): $H$ is isomorphic to the generalized Jacobian of $\tilde{C}^{\prime}$ corresponding to the modulus $E_{0}$ and

- Example 1 in [35]: the generalized Jacobian of $\tilde{C}^{\prime}$ corresponding to the modulus $E_{0}$ is none other than $A_{\tilde{C}}^{0}$.

Notice that $F$ and $E$ (similarly $E_{0}$ and $F_{0}$ ) are disjoint.

Put $D^{\prime}:=\operatorname{Ker}\left(D_{F_{0}} \rightarrow D_{F}\right)$; there is a natural map $D^{\prime} \stackrel{\mu}{\rightarrow} A_{\tilde{C}}$ induced by the inclusion of $F_{0}$ in $\tilde{C}$. We set $D:=\operatorname{Ker}\left(D^{\prime} \stackrel{\mu}{\rightarrow} A_{\tilde{C}} \rightarrow D_{\tilde{C}}\right)$, the last being 
the group of connected (= irreducible) components of $\tilde{C}$. The group $D(\bar{S})$ is generated by differences $(x-y) \in D_{F_{0}}(\bar{S})$ such that $b_{0}(x)=b_{0}(y) \in F(\bar{S})$ and $x, y$ are in the same geometric component of $\tilde{C}$.

Using Theorem 6.5, we may define $h_{1}(C)$ [21].

Definition 6.6. (Lichtenbaum) The homological 1-motive $h_{1}(C)$ is the 1 motive

$$
\left[D \stackrel{\mu}{\rightarrow} A_{\tilde{C}}^{0}\right] .
$$

The key identification is in the

Theorem 6.7. One has natural isomorphisms

(i) $H_{m}^{1}(C)(1) \stackrel{\sim}{\rightarrow} M^{1}\left(C_{\bullet}\right)$.

(ii) $h_{1}(C) \stackrel{\sim}{\rightarrow} M_{1}\left(C_{\bullet}\right)$.

Proof. We begin with (i). We have defined $R$ to be the subgroup scheme of $\underline{\mathbb{Z}}^{w\left(E_{0}\right)}$ corresponding to the intersection of the kernels of $\underline{\mathbb{Z}}^{w\left(E_{0}\right)} \rightarrow$ $\pi_{0}\left(P i c_{C_{0}^{\prime}}^{0}\right)$ and $\underline{\mathbb{Z}}^{w\left(E_{0}\right)} \stackrel{\delta_{0}^{*}-\delta_{1}^{*}}{\longrightarrow} \underline{\mathbb{Z}}^{w\left(E_{1}\right)}$.

It is a simple consequence of the definition of $C_{\bullet}^{\prime}$ that we have a complex:

$$
\underline{\mathbb{Z}}^{w(E)} \stackrel{c^{*}}{\longrightarrow} \underline{\mathbb{Z}}^{w\left(E_{0}\right)} \stackrel{\delta_{0}^{*}-\delta_{1}^{*}}{\longrightarrow} \underline{\mathbb{Z}}^{w\left(E_{1}\right)} .
$$

But $c^{*}$ is an isomorphism (22). Therefore, $R=\operatorname{Ker}\left(\underline{\mathbb{Z}}^{w\left(E_{0}\right)} \rightarrow \pi_{0}\left(\operatorname{Pic} C_{C_{0}^{\prime}}^{0}\right)\right)$. Since the group of connected components of the Picard schemes of $C^{\prime}$ and $C_{0}^{\prime}=\tilde{C}^{\prime}$ are isomorphic ([3], Proposition 10, $\S 9.2$, p.248-9), we find an isomorphism $c^{*}: \operatorname{Ker}(\alpha) \stackrel{\stackrel{\sim}{\longrightarrow}}{\rightarrow} R$.

The diagram below is commutative:

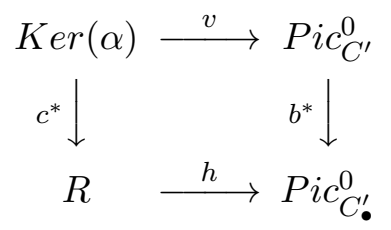

with isomorphisms (see Lemma 6.4) for vertical arrows. This proves (i).

We remark that $C_{0}:=\tilde{C}$ and $C_{1}:=\tilde{C} \times_{C} \tilde{C}$. As usual, we write the Albanese 1-motive of $C \bullet$ as $[N \stackrel{\partial}{\rightarrow} G]$ (see $\S$ 团).

The Albanese 1-motive of a special scheme $W$ is the neutral component of the Albanese scheme $A_{W}$ of $W$ (2.1). Any normal curve is a special scheme. It is clear from the definitions of the Albanese 1-motive and the homological 1-motive that, for a normal curve $Y$, one has $h_{1}(Y)=M_{1}(Y)=A_{Y}^{0}$.

Returning back to our curve $C$ and $C_{\bullet}$, let us set $A_{i}:=A_{C_{i}}$. Since each $C_{i}$ is the disjoint union of the diagonal (isomorphic to $\tilde{C}$ ) and a finite étale scheme $\left.X_{i}(i \geq 1)\right)^{9}$, we have that $A_{i}=A_{\tilde{C}} \times_{S} D_{X_{i}}$. We have $A_{0}=A_{\tilde{C}}$. Therefore, the simplicial large group $A_{\bullet}$ contains a constant simplicial large group $B_{i}:=A_{\tilde{C}}$ (for all non-negative integers $i$ ). Viewing these simplicial groups (and $D_{X_{i}}$ ) as complexes (by normalization), we see

\footnotetext{
${ }^{9}$ This is because $C_{0}=\tilde{C}$.
} 
(1) that $A_{\tilde{C}}=H_{0}\left(B_{\bullet}\right)=H_{0}\left(A_{\bullet}\right)$.

(since $C_{0}=\tilde{C}$ ) that $\pi_{0}\left(A_{0}\right)=D_{\tilde{C}}$. This in turn implies that

$$
N=\frac{\operatorname{Ker}\left(D_{X_{1}} \stackrel{\delta_{0}-\delta_{1}}{\longrightarrow} D_{\tilde{C}}\right)}{\operatorname{Im}\left(D_{X_{2}} \stackrel{\delta_{0}-\delta_{1}+\delta_{2}}{\longrightarrow} D_{X_{1}}\right)} .
$$

In particular, we deduce $G=A_{\tilde{C}}^{0}$. So we have already proved that $W_{-1} h^{1}(C)=W_{-1} M^{1}\left(C_{\bullet}\right)$. We will use the previous description of $N$ to show that $D$ is actually $N$ (compatible with the map $\mu$ ) and this will finish the proof of (ii).

Let us put $F_{n}$ to be the $(n+1)$-fold fibre product of $F_{0}$ over $F$. We have $F_{1}:=F_{0} \times{ }_{F} F_{0}$ and $F_{2}:=F_{0} \times_{F} F_{0} \times_{F} F_{0}$.

The group $D_{X_{1}}(\bar{S})$ is generated by points $(x, y) \in F_{1}(\bar{S})$ with $x \neq y$ (i.e. $x, y$ are distinct points of $\left.F_{0}\right)$. By definition, the points $x, y \in \tilde{C}(\bar{S})$ satisfy $b_{0}(x)=b_{0}(y) \in C(\bar{S})$. Set $K:=K \operatorname{Ker}\left(D_{X_{1}} \stackrel{\delta_{0}-\delta_{1}}{\longrightarrow} D_{\tilde{C}}\right)$. The group $K(\bar{S})$ is generated by points $(x, y) \in F_{1}(\bar{S})$ which map to the same (geometrically) connected component of $\tilde{C}$. There is a natural morphism $\gamma: K \rightarrow D$, which we describe on the level of the geometric points: we send $(x, y)$ to the element $x-y \in D(\bar{S})$. The morphism $\gamma$ is surjective (by the seminormality of $C$ ).

Let us turn to the group $D_{X_{2}}(\bar{S})$. It is generated by elements $(x, y, z) \in$ $F_{2}(\bar{S})$ with $x \neq y, y \neq z, x \neq z$. By definition, we have $b_{0}(x)=b_{0}(y)=$ $b_{0}(z) \in C(\bar{S})$. One has a map

$$
\delta: D_{X_{2}} \stackrel{\delta_{0}-\delta_{1}+\delta_{2}}{\longrightarrow} K \quad(x, y, z) \mapsto(y, z)-(x, z)+(x, y) .
$$

The composition $\gamma . \delta$ can be checked to be zero; in fact, $\operatorname{Ker}(\gamma)=\operatorname{Im}(\delta)$. Since the group $N$ is $\operatorname{Coker}(\delta)$, we see that $N=D$. It is clear that the maps from $N$ and $D$ to $A_{\tilde{C}}^{0}$ are compatible with this isomorphism. This proves (ii).

Theorem 6.8. (Lichtenbaum, unpublished) The 1-motives $H_{m}^{1}(C)(1)$ and $h_{1}(C)$ are dual.

Proof. This follows from Theorems 6.7 and 5.1.

Corollary 6.9. (Hodge Realization) For a curve $C$ over $\mathbb{C}$, one has an isomorphism

$$
H_{1}(C) \cong \mathfrak{T}\left(h_{1}(C)\right) \text {. }
$$

Proof. Combine Theorems 10.3.8 of [6] and 5.1.

The motivic $H^{1}$ of $C$ can also be interpreted as the motivic relative cohomology group $H_{m}^{1}\left(\tilde{C}^{\prime}-E_{0}\right.$ rel $\left.F_{0}\right)(1)$. Likewise, one can interpret $h_{1}(C)$ as the motivic relative homology group $H_{1}^{m}\left(\tilde{C}^{\prime}-F_{0}\right.$ rel $\left.E_{0}\right)$. With these identifications, the duality theorem translates to a Lefschetz duality theorem 
$H_{m}^{1}\left(\tilde{C}^{\prime}-E_{0}\right.$ rel $\left.F_{0}\right)(1) \stackrel{\sim}{\rightarrow}\left(H_{1}^{m}\left(\tilde{C}^{\prime}-F_{0} \text { rel } E_{0}\right)\right)^{*}$ (see 1.5 of [队] $)$. The resulting duality of the Hodge realizations is a classical result 泊, 42 (especially p. 390-92).

\section{1-MOTIVES ASSOCIATED WITH VARIETIES}

Let $Y$ be a scheme over $S$. By de Jong [10], there exists a diagram

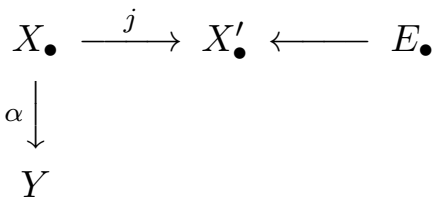

where the smooth simplicial scheme $X_{\bullet}$ is the open complement of a reduced simplicial divisor with normal crossings $E_{\bullet}$ in a smooth projective simplicial scheme $X_{\bullet}^{\prime}$ such that, via $\alpha, X_{\bullet}$ is a proper hypercovering of $Y$. Using this, it is possible (as in [6] §6.3) to translate the theory developed for simplicial schemes such as $X$ • to obtain invariants for the scheme $V$. This has been treated in 30.

\section{REFERENCES}

[1] L. Barbieri-Viale, V. Srinivas. Albanese and Picard 1-motives. C. R. Acad. Sci. Paris Sér. I Math. 326 (1998), no. 12, 1397-1401.

[2] L. Barbieri-Viale, V. Srinivas. Albanese and Picard 1-motives. Preprint (June 1999), http://www.math.uiuc.edu/K-theory/0352

[3] S. Bosch, W. Lutkeböhmert, M. Raynaud. Néron Models. Springer Verlag. 1990.

[4] C. Chevalley. Introduction to the theory of algebraic functions in one variable. Math. Surv., VI, AMS, New York, 1951.

[5] P. Colmez. Integration sur les variétés p-adiques. Astérisque 248 SMF 1998.

[6] P. Deligne. Théorie de Hodge II. Publ. IHES. 40 (1972) 5-58; III. Publ. IHES. 44 (1974) 3-77.

[7] P. Deligne. Poids dans la cohomologie des variétés algébriques. Actes du ICM. I. pp. 79-85, Vancouver, 1974.

[8] P. Deligne. A quoi servent les motifs? Proc. Symp. Pure Math. 55 pp. 143-161, AMS, Providence RI., 1996.

[9] M. Demazure and P. Gabriel. Introduction to algebraic geometry and algebraic groups. North-Holland-Amsterdam 1980.

[10] A. J. de Jong. Smoothness, semistability and alterations. Publ. IHES. 83 (1996), 51-93.

[11] H. Esnault, V. Srinivas, E. Viehweg. The universal regular quotient of the Chow group of points on projective varieties. Invent. Math. 135 (1999), no. 3, 595-664.

[12] G. Faltings, G. Wüstholz. Einbettungen kommutativer algebraischer Gruppen und einige ihrer Eigenschaften. J. Reine. Angew. Math 354 (1984) pp. 175-205.

[13] H. Gillet. Universal cycle classes. Compositio Mathematica 49 (1983) pp. 3-49.

[14] M. Goresky and R. MacPherson. Stratified Morse theory. Ergebnisse der Mathematik und ihrer Grenzgebiete (3), 14, Springer-Verlag, Berlin-New York, 1988.

[15] P. Griffiths and J. Harris. Principles of algebraic geometry. Pure and Applied Mathematics. Wiley-Interscience, New York, 1978.

[16] A. Grothendieck. Technique de descent et théorèmes d'existence en géométrie algébrique VI. Séminaire Bourbaki no. 236. (1961/62). 
[17] R. Hartshorne. Algebraic Geometry. Graduate Texts in Mathematics 52, Springer New-York 1977.

[18] L. Illusie. Complexe de de Rham-Witt et cohomologie cristalline. Ann. Sci. École Norm. Sup. (4) 12 (1979), no. 4, 501-661.

[19] L. Illusie. Crystalline cohomology, Proc. Symp. Pure Math. 55 pp. 43-70, AMS, Providence RI., 1994.

[20] U. Jannsen. Motivic sheaves and filtrations on Chow groups . Motives (Seattle, WA, 1991), 245-302, Proc. Sympos. Pure Math., 55, Part 1, Amer. Math. Soc., Providence, RI, 1994.

[21] S. Lichtenbaum. Suslin homology and Deligne 1-motives. Algebraic K-theory and algebraic topology (Lake Louise, AB, 1991), NATO Adv. Sci. Inst. Ser. C Math. Phys. Sci., 407, Kluwer Acad. Publ., Dordrecht, (1993) 189-196.

[22] S. Lichtenbaum. Quasi-motives of curves. Algebraic K-theory (Toronto, ON, 1996), Fields Inst. Commun., 16, Amer. Math. Soc., Providence, RI (1997) 185-197.

[23] J. P. May. Simplicial objects in algebraic topology. Reprint of the 1967 original. Chicago Lectures in Mathematics. University of Chicago Press, Chicago, IL, 1992.

[24] B. Mazur and W. Messing. Universal extensions and one dimensional crystalline cohomology. Lecture Notes in Mathematics 370, Springer-Verlag, Berlin-New York, 1974.

[25] W. Messing. The universal extension of an abelian variety by a vector group. Symposia Mathematica (Convegno di Geometria, INDAM, Rome, 1972) XI. Academic Press, London (1973), pp. 359-372.

[26] J. S. Milne. Étale cohomology. Princeton University Press 1980.

[27] H. Önsiper. Rigidified Picard functor and extensions of abelian schemes. Arch. Math. 49 (1987) pp. 503-507.

[28] F. Oort. Commutative group schemes. Lecture Notes in Mathematics 15, SpringerVerlag, 1966.

[29] N. Ramachandran. Mixed motives of schemes. Doctoral Dissertation. Brown University (May 1996).

[30] N. Ramachandran. A conjecture of Deligne on one-motives. Preprint. (June 1998) math.AG/9806114.

[31] M. Rapoport, N.Schappacher and P. Schneider (eds.). Beilinson's conjectures on special values of L-functions. Perspectives in Mathematics 4. Academic Press 1988.

[32] M. Rosenlicht. Generalized Jacobian varieties. Ann. of Math. 59 (1954) pp.505-530.

[33] M. Rosenlicht. A universal mapping property of generalized Jacobians. Amer. J. of Math. 66 (1957) pp. 80-88.

[34] J.-P. Serre. Morphismes universels et variété d'Albanese. Variétés de Picard. Séminaire C. Chevalley. E.N.S. Paris, 1958-59.

[35] J.-P. Serre. Morphismes universels et différentiels de troisième éspèce. Variétés de Picard. Séminaire C. Chevalley. E.N.S. Paris, 1958-59.

[36] J.-P. Serre. Algebraic groups and class fields. Graduate text in Mathematics. 117 Springer Verlag, New York-Berlin, 1988.

[37] J.-P. Serre. email to the author. May 16, 1995.

[38] A. Grothendieck et al. Groupes de monodromie en géométrie algébrique. Lecture notes in Mathematics 288, Springer Verlag, 1972-3.

[39] R. G. Swan. On seminormality. J. Algebra 67 (1980), no. 1, 210-229.

[40] V. Voevodsky. Homology of schemes. Selecta Math.(N.S.) 2 (1996), pp. 111-153.

[41] A. Weil. Variétés abéliennes. Colloque d'algèbre et théorie des nombres, CNRS, pp. 125-128. Paris. 1949 (= Collected Papers [1950a]).

[42] A. Weil. Review of Introduction to the theory of algebraic functions of one variable, by C. Chevalley, B.A.M.S. 57, (1951) pp. 384-398 (= Collected Papers [1951c]). 
Department of Mathematics, University of Maryland, College Park, MD 20742-4015, USA

E-mail address: atma@math.umd.edu 